\title{
Diversity and Distribution of Russula in India with Reference to Central Indian species
}

\author{
R.K. Verma ${ }^{1}$, Vimal Pandro ${ }^{1 *}$ and Abhishek Pyasi ${ }^{2}$ \\ ${ }^{1}$ Forest Pathology Discipline, Forest Protection Division, Tropical Forest Research Institute, \\ Jabalpur - 482 021, Madhya Pradesh, India \\ ${ }^{2}$ Dayanand Anglo Vedic Public School, Burhar-484110, Shahdol, Madhya Pradesh, India \\ *Corresponding author
}

\section{A B S T R A C T}

\begin{tabular}{|l|}
\hline Ke y w o r d s \\
$\begin{array}{l}\text { Agaricomycetes, } \\
\text { Distribution, Ecto- } \\
\text { mycorrhiza, } \text { Russula, Sal } \\
\text { forest }\end{array}$ \\
\hline Article Info \\
\hline $\begin{array}{l}\text { Accepted: } \\
\text { 24 September } 2018 \\
\text { Available Online: } \\
\text { 10 October } 2018\end{array}$ \\
\hline \hline
\end{tabular}

An account of mushrooms belonging to genus Russula reported from different part of India is given. Total 124 species of the genus were compiled from literature with their records of habitat, distribution and references. Russula species were recorded from 13 states of India. Uttarakhand represent the maximum diversity of Russula species (57) followed by Kerala (17), Himachal Pradesh (13), West Bengal (13), Jammu and Kashmir and Sikkim 10 species each, Madhya Pradesh (6) and Nagaland (6). Other states from where species recorded include Meghalaya (3), Punjab (2) while Maharashtram and Tamil Nadu are represented by only one species each. Six species of Russula namely: $R$. adusta, $R$. cinerella, $R$. congoana, $R$. delicula, $R$. leelavathyi and $R$. michiganensis were recorded and described for the first time from Sal forest of central India (Madhya Pradesh and Chhattisgarh). These fungi are known to form ectomycorrizal association with sal trees. Among them some species are edible and sold in the local market during rainy season.

\section{Introduction}

Members of the Russula ceae are characterized by their fleshy and often brightly coloured fruiting bodies with prominent lamellae, a heteromerous context traversed by conducting hyphae, warted, light-colored amyloid basidiospores which exhibit various types of ornamentations. Russula ceae was established by Roze in 1876 and it is one of the largest ectomycorrhizal families. The members of this family form ectomycorrhizal association in different angiosperm and gymnosperm trees like Abies, Acer, Betula, Diospyros, Hopea, Larix, Myristica,
Rhododendron, Shorea, Tsuga, Vateria, etc. The genus is represented by about 130 taxa from India (Das et al., 2014). North-western subtropical and temperate forests of Himalayan region of Uttarakhand state are represented most Russula species. There is a large gap that exists with respect to knowledge diversity of macro-fungi in India. For proper planning and management of forests and the conservation of their biodiversity, characterization, systematics and ecology of these macro-fungi is essential. Many Russula species for example, $R$. crustosa, $R$. lutea, $R$. olivacea, $R$. parvovirescens, $R$. senecis, $R$. virescens are edible (Atri et al., 2010; Das et 
al., 2002; Bhatt and Lakhanpal, 1988a, b; Kalita et al., 2016; Khatua et al., 2015; Semwal et al., 2014). The present paper provides a comprehensive database of diversity of Russula species in India. Sixspecies of Russula from central India are alsodescribed. Edible species are also listed.

\section{Materials and Methods}

Specimens of mushrooms were collected from Madhya Pradesh and Chhattisgarh during rainy seasons. Some parts of collected samples were preserved in $70 \%$ alcohol just after collection for microscopic study. The fruit bodies of fungi were dried under the sun or in the wooden box lighted with $100 \mathrm{~W}$ electric bulb. Microscopic slides were prepared by using stain, mountant, clearing and softening chemicals. Slides were observed under advanced research microscope (Leica, Germany) using 5x, 10x, 20x, 40x objectives and $10 x$ and $15 x$ eyepieces.

Observations under phase contrast and dark field were also made whenever required. Photomicrography was done with the help of a digital camera (make, Leica) attached to the advanced microscope. Identification of fungi has been done with the help of published literature, monographs, books, keys, etc. (Abraham et al., 1980; Atri and Kour, 2003; Atriand Saini, 1986, 1990a; 1990b; 1990c; Atri et al., 1992, 1997, 2016; Berkeley, 1851, 1856, 1876; Bhatt et al., 1995, 2007; Buyckand Atri, 2011; Chaudharyand Tripathy, 2016; Crouset al., 2016; Das, 2013; Das and Sharma, 2001, 2003, 2005b; Das et al., 2002c; 2005a, 2006, 2008; 2010; 2013a,b; 2014; 2017; Dhancholia, 2011; Duttaet al., 2015; Farooket al., 2013; Ghosh and Das, 2017; Hedawoo, 2010; Joshi et al., 2012; Kauret al., 2011; Kumar et al., 2014; Manimohanand Deepna, 2011; Mohanan, 2011, 2014; Natarajanand Raman, 1983; Pavithra et al., 2017; Pradeep and Vrinda, 2007, 2010;
Rawla, 2001; Rawla and Sarwal, 1983; Romagnesi, 1945; Saini et al., 2010; Saini and Atri, 1981, 1984, 1989a, b; Saini et al., 1988, 1989; Sarwal, 1984; Sathe et al., 1980; Shaffer, 1962; Shajahan and Samajpati, 1995; Sharma and Das, 2002; Varghese et al., 2010; Vishwakarma et al., 2012; Vrinda et al., Vrinda 1997a, b; Watling and Gregory, 1980))

\section{Results and Discussion}

\section{Taxonomic description}

\section{Russula adusta (Pers.) Fr. (Figures 1-4)}

$$
\text { = Agaricusadustus Pers. = Omphaliaadusta }
$$
Pers.

Basidiome medium to large sized. Pileus 5$8 \mathrm{~cm}$ diam, with a deep depression at centre at maturity; margin in rolled when young, becoming uplifted when fully expanded; surface dull white becoming grayish white then dark brown and finally black on handling or aging, non-striate. Lamella adnate, white turning black on handling, more or less crowded with lamellulae of different lengths, sometimes bifurcated close to the stipe; edge smooth, entire. Stipe 4-6 x 1-2cm, central equal, solid; surface chalky white, immediately turning black when handled, odour not distinctive. Context white discolouring blackish on exposure, heteromerous with nests of sphaerocytes. Spores 6-8 x 5-7 $\mu \mathrm{m}$ globose to broadly ovoid, strongly amyloid with an ornamentation of prominently verrucose and connective forming a partial reticulum; supra-hilarplageinamyloid. Basidiaclavate 31-46 × 7-8 $\mu \mathrm{m}$, tetrasporate. Lamella-edge sterile with croweded macrocystidia 59-80 x 6-8 $\mu \mathrm{m}$, clavate, mucronate with oleaginous refractive contents discolouring black, pleurocystidia similar. Haymenophoraltrama heteromorphous with sphaerocytes. Pileipellis an exocutis devoid of dermatocystidia. Caulocystidia, 30-45x5-7 $\mu \mathrm{m}$. 


\section{Collection examined}

Mycorrhizal on sal (Shorearobusta), Amarkantak-Achanakmar Biosphere Reserve, Madhya Pradesh and Chhattisgarh, 24/07/2012, Mycology Herbarium, Tropical Forest Research Institute, Jabalpur TF 2789.

\section{Russula cinerella Pat. (Figures 5-7)}

Basidiomesare small to medium sized, pileus $4-7 \mathrm{~cm}$ diam, convex to applanate, depressed at the centre, surface light grey to brownish orange, silky, dry smooth, margin undulate and incurved. Lamellae adnate, white becoming pale yellowish, crowded up to $5 \mathrm{~mm}$ wide. Stipe 4-6 x 1.0-1.4cm, cylindric, solid; surface white. Context up to $5 \mathrm{~mm}$ thick, white becoming pinkish brown on exposure. Sporeprint pale cream. Spores 5-8 x 5-7.5 $\mu \mathrm{m}$, subglobose to ovoid, hyaline, ornamented with

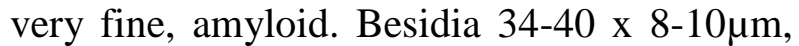
clavate, bearing 4 sterigmata. Lamella-edge heteromorphous. Cheilocystidia 27-35 x 10$12 \mu \mathrm{m}$, cylindric to fusoid, often apically constricted or capitates, hyaline, with numerous refractive contents. Pleurocystida 31-41 x $8-9 \mu \mathrm{m}$, sinuous, lanceolatefusoid, often constricted or mucronate, with abundant granular contents. Hymenophoraltrama irregular and intermixed Pileal surface a disrupted trichodermium subtended by a broad hypodermium. Trichodermium of more or less erect hyphae,2-3.5 $\mu \mathrm{m}$ diam., sometimes agglutinated into fascicles; dermatocystidia absent. Hypodermium 110-130 $\mu \mathrm{m}$ thick, of tightly interwoven hyphae, slightly agglutinated.

\section{Collection examined}

Mycorrhizal on sal, Amarkantak-Achanakmar Biosphere Reserve, Madhya Pradesh and Chhattisgarh, 24/07/2012. Mycology Herbarium, Tropical Forest Research Institute, Jabalpur TF 2788.

\section{Russula congoana Pat. (Figures 8-11)}

Basidiome small to medium sized.Pileus 4-5.5 $\mathrm{cm}$. diam, at first convex then expanded with slight central depression; surface uniformely pastel red to red, smooth finely striate at the margin, sticky. Lamellae adnate, white to pale cream exceeding the gills, up to $5 \mathrm{~mm}$ wide, close; edge entire, concolourous to the sides. Context thin white. Stipe $2.5-3.5 \mathrm{~cm} \times 7-12$ $\mathrm{mm}$, central cylindrical equal or slightly broader at the base, solid becoming stuffed and hollow surface creamy white with a pinkish fint at the base, smooth. Odour pleasant, test met distinctive. Spores 4-6 x 3$5 \mu \mathrm{m}$, subglobose ellipsoid to oblong, densely ornamented with coarse, amyloid, verrucose interconnected by a reticulate system. Basidiaclavate, 29-39 x 10-14 $\mu \mathrm{m}$, 4-spored. Macrocystidia scattered on both edges and

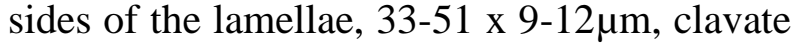
to fusiform, frequently mucronate, thin walled. Subpellis slightly gelatinized. Suprapellis composed of eract, shortly cylindrical element, 3-5diam. Pileocystidia numerous,

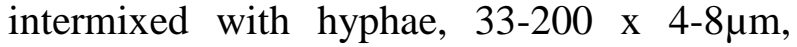
cylindrical clavate to fusiform, obtuse or slightly constricted to fusiform, obtuse or slightly constricted at large apex. Clamp connection absent.

\section{Collection examined}

Mycorrhizal withsal, AmarkantakAchanakmar Biosphere Reserve, Madhya Pradesh and Chhattisgarh, 24/07/2012, Mycology Herbarium, Tropical Forest Research Institute, Jabalpur, TF2790.

\section{Russula delicula Romagn. (Figures 12-14)}

Bisidiome small to medium sized. Pileus 4.5$5.5 \mathrm{~cm}$ diam, convex becoming uplifted and infundibula form at maturity margin inrolled when young, becoming uplifted when fully expanded; surface yellowish grey with grayish 
orange tints, viscid when wet, otherwise dry, non-striate. Lamellae subdecurrent, white, up to $4 \mathrm{~mm}$ broad, often forked at or near the stipe, interveinose, white to pale buff, close, without lamellulae; edge smooth, entire. Context white. Stipe 15-32 x 1.2-2 mm; central, equal or slightly attenuated below, solid; surface white, smooth, annulus absent.

Odour not distinctive Spores 5.0-8.75 x 5.0$7.5 \mu \mathrm{m}$, globose to broadly avoid strongly amyloid with an ornamentation of moderately large verrucose \& thin connectives forming a partial to complete reticulam. Basidia 36-41 x $4-7 \mu \mathrm{m}$, clavate, tetrasporate. Cystidia scattered both on edges \& sides of the lamellae, 78-112 X 15-26 $\mu \mathrm{m}$ clavate-fusoid sometimes with a long, attenuated neck bearing a small bulk apically, filled with oleaginous refractive contents. Subhymenium psendoparenchymatous. Hymenophoraltrama heteromorphous pileipellis with horizontally arranged non-gelatinized hyphre, devoid of dermatocystidia.

\section{Collection examioned}

Mycorrhizal on sal, Amarkantak-Achanakmar Biosphere Reserve, Madhya Pradesh and Chhattisgarh, 24/07/2012, Mycology Herbarium, Tropical Forest Research Institute, Jabalpur, TF 2792.

\section{Russula leelavathyi K.B. Vrinda, C.K. Pradeep \& T.K. Abraham (Figures 15-17)}

Basidiome small to medium sized pilus $3-5 \mathrm{~cm}$ diam., fleshy, convex than expanded with a central depression; surface uniformely ivory to grayish white, areolately cracked forming patches of ivory squamules on a off white ground, entire at the disk; margin radially plicato-striate for two third of radium from the margin and cracking along radial striae to expose underlying white context below, gelatinized under wet weather. Lamellae adnexed to subdecurrent, white up to $3 \mathrm{~mm}$ white, heteromerous with thin walled hyaline hyphae 2-6mm wide intermixed with sphaerocytes $17-23 \mu \mathrm{m}$. Stipe $3-5 \mathrm{~cm}$ x 5$13 \mathrm{~mm}$, central, cylindrical, equal, solid, becoming stuffed; surface white, smooth. Basidiopores 7.5-6.25 x 7.5-7.5 $\mu \mathrm{m}$, subglobose to broadly ovoid, hyaline with an ornamentation of coarse, amyloid, verrucose and scattered fine connectives forming a pilus reticulum. Basidiaclavate 45-56 x 7-9 $\mu \mathrm{m}$, tetraspored.

Lamella edge sterile, cheilocystidia 33-35 x 9$10 \mu \mathrm{m}$, clavate, fusoid, lageniform often with actually pointed or mucronate apex. Macrocystidia 73-87 x 4.7-6.5 $\mu \mathrm{m}$, ventricose, fusoid to acuminate, thin-walled, with granular contents, numerous both on sides and edges of the lamellae. Hymenophoraltrama hateromerous composed of thin-walled, hyaline hyphae, intermixed with sphaerocytes. Piliepellis distinctly two layered an upper epithelial layer and a lower loosely interwoven gelatinized layer. Stipitipellis with abundant caulocystedia $20-40 \times 7-12 \mu \mathrm{m}$ similar to cheilocystedia. The furcated lamellae together with lack of lamellulae are characteristic feature of this species.

\section{Collection examined}

Mycorrhizal on sal, Amarkantak-Achanakmar Biosphere Reserve, Madhya Pradesh and Chhattisgarh, 24/07/2012, Mycology Herbarium, Tropical Forest Research Institute, Jabalpur, TF 2793.

\section{Russula michiganensis Shaffer (Figures 18- 20)}

Sporocarp small to medium sized. Pileus up to $2.5-6 \mathrm{~cm}$ diam, convex to broadly convex with a central depression becoming uplifted in older ones; surface grayish brown becoming black on aging, non-striate. 
Table.1 Distribution of Russula mushroom in India

\begin{tabular}{|c|c|c|c|c|}
\hline $\begin{array}{l}\text { S. } \\
\text { N. }\end{array}$ & Name of fungus & Habit & Distribution & Reference \\
\hline 1. & $\begin{array}{l}\text { Russula abbotensisK. } \\
\text { Das \& J.R. Sharma }\end{array}$ & - & $\begin{array}{l}\text { From Abbot Mount, } \\
\text { Champawat, } \\
\text { Uttarakhand }\end{array}$ & $\begin{array}{l}\text { Das and } \\
\text { Sharma } \\
\text { (2005a) }\end{array}$ \\
\hline 2. & $\begin{array}{l}\text { Russula aciculocystis } \\
\text { Kauffman ex Bills \& } \\
\text { O.K. Mill. }\end{array}$ & - & $\begin{array}{l}\text { Thiruvananthapura } \\
\text { m, Kerala }\end{array}$ & $\begin{array}{l}\text { Pradeep and } \\
\text { Vrinda } \\
(2010) ; \\
\text { Mohanan } \\
(2011,2014)\end{array}$ \\
\hline 3. & Russula adusta(Pers.) Fr. & $\begin{array}{l}\text { in leaf litter and } \\
\text { ectomycorrhizal } \\
\text { with Vateriaindica }\end{array}$ & $\begin{array}{l}\text { From Kailana, } \\
\text { Chakrata, } \\
\text { Mayawati, } \\
\text { Jageshwar, } \\
\text { Uttarakhand; } \\
\text { Malappuram and } \\
\text { Thiruvananthapura } \\
\text { m, Kerala and } \\
\text { Mangalore, } \\
\text { Karnataka }\end{array}$ & $\begin{array}{l}\text { Saini and } \\
\text { Atri (1984); } \\
\text { Atri and } \\
\text { Saini } \\
\text { (1990b); Das } \\
\text { and Sharma } \\
\text { (2005b);Prad } \\
\text { eep and } \\
\text { Vrinda, } \\
\text { (2010);Moha } \\
\text { nan (2011, } \\
\text { 2014); } \\
\text { (Pavithra et } \\
\text { al., (2017) }\end{array}$ \\
\hline 4. & $\begin{array}{l}\text { Russula } \\
\text { aerugineaLindblad:-Fr. }\end{array}$ & - & $\begin{array}{l}\text { Puliebzie, Zakhama } \\
\text { and Pherma forest } \\
\text { ranges, Nagaland }\end{array}$ & $\begin{array}{l}\text { Kumar et al., } \\
\text { (2014) }\end{array}$ \\
\hline 5. & $\begin{array}{l}\text { Russula } \\
\text { alachuanaMurrill }\end{array}$ & - & $\begin{array}{l}\text { Kailana, Chakrata, } \\
\text { Uttarakhand }\end{array}$ & $\begin{array}{l}\text { (Atri, Saini, } \\
\text { 1986). }\end{array}$ \\
\hline 6. & Russula albidaPeck & 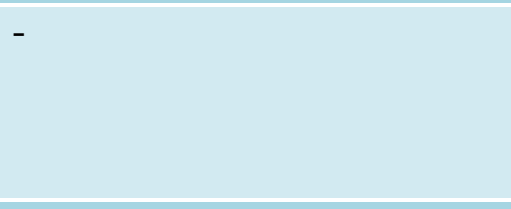 & $\begin{array}{l}\text { Nagdev-Jhandidhar } \\
\text { Forest, Punjab and } \\
\text { Khirsu Forest, } \\
\text { Uttarakhand }\end{array}$ & $\begin{array}{l}\text { Bhatt et al., } \\
\text { (1995) }\end{array}$ \\
\hline 7. & $\begin{array}{l}\text { Russula } \\
\text { albonigra(Krombh.) Fr. }\end{array}$ & $\begin{array}{l}\text { Ectomycorrhizal on sal in sal } \\
\text { forest }\end{array}$ & $\begin{array}{l}\text { Deoban, Chakrata, } \\
\text { Uttarakhand, } \\
\text { Gidhani and } \\
\text { Jhargram, West } \\
\text { Bengal }\end{array}$ & $\begin{array}{l}\text { Saini and } \\
\text { Atri (1984); } \\
\text { Atri and } \\
\text { Saini (1986, } \\
\text { 1990a);Shaja } \\
\text { han and } \\
\text { Samajpati } \\
\text { (1995) }\end{array}$ \\
\hline 8. & $\begin{array}{l}\text { Russula alnetorum } \\
\text { Romagnesi }\end{array}$ & $\begin{array}{l}\text { in leaf litter in the forest } \\
\text { range }\end{array}$ & $\begin{array}{l}\text { Mankoi and } \\
\text { Chungtia, Nagaland }\end{array}$ & $\begin{array}{l}\text { Kumar et al., } \\
\text { (2014) }\end{array}$ \\
\hline 9. & Russula alutacea (Fr.) & on wood & Gulmarg, Jammu & Berkeley \\
\hline
\end{tabular}




\begin{tabular}{|c|c|c|c|c|}
\hline & $\begin{array}{l}\text { Fr. } \equiv \text { Agaricus alutaceus } \\
\text { Fr., }\end{array}$ & & and Kashmir & (1876) \\
\hline 10. & Russula amoenaQuél. & - & $\begin{array}{l}\text { Palakkad, Kollam, } \\
\text { Wayanad, and } \\
\text { Thiruvananthapura } \\
\text { m, Kerala }\end{array}$ & $\begin{array}{l}\text { Vrinda et al., } \\
(1997 c) ; \\
\text { Pradeep and } \\
\text { Vrinda } \\
\text { (2010; } \\
\text { Mohanan } \\
\text { (2011) }\end{array}$ \\
\hline 11. & $\begin{array}{l}\text { Russula amoenicolor } \\
\text { Romagn. }\end{array}$ & - & $\begin{array}{l}\text { JabbarKhet, } \\
\text { Mussoorie, } \\
\text { Uttarakhand }\end{array}$ & $\begin{array}{l}\text { Rawla and } \\
\text { Sarwal } \\
\text { (1983). }\end{array}$ \\
\hline 12. & $\begin{array}{l}\text { Russula amoenicolorvar. } \\
\text { Ramgarhensis K. Das, } \\
\text { J.R. Sharma \& R.P. } \\
\text { Bhatt }\end{array}$ & - & $\begin{array}{l}\text { Ramgarh, Nainital, } \\
\text { Uttarakhand }\end{array}$ & $\begin{array}{l}\text { Das et al., } \\
\text { (2005a). }\end{array}$ \\
\hline$\overline{13 .}$ & $\begin{array}{l}\text { Russula amoenolens } \\
\text { Romagn. }\end{array}$ & $\begin{array}{l}\text { From Nagdev-Jhandidhar } \\
\text { Forest }\end{array}$ & Khirsu, Uttarakhand & $\begin{array}{l}\text { Bhatt et al., } \\
\text { (1995) }\end{array}$ \\
\hline$\overline{14 .}$ & $\begin{array}{l}\text { Russula anatine } \\
\text { Romagn. }\end{array}$ & & $\begin{array}{l}\text { DafiaDhura, } \\
\text { Pithoragarh; } \\
\text { Dhakuri, } \\
\text { Bageshwar, } \\
\text { Uttarakhand }\end{array}$ & $\begin{array}{l}\text { Das and } \\
\text { Sharma } \\
\text { (2003) }\end{array}$ \\
\hline$\overline{15 .}$ & $\begin{array}{l}\text { Russula appendiculata } \\
\text { K. Das, S.L. Mill. \& J.R. } \\
\text { Sharma }\end{array}$ & Associated with Pinus & $\begin{array}{l}\text { Gagar, Nainital, } \\
\text { Uttarakhand }\end{array}$ & $\begin{array}{l}\text { Das et al., } \\
\text { (2006a) }\end{array}$ \\
\hline 16. & $\begin{array}{l}\text { Russula arunii S. Paloi, } \\
\text { A.K. Dutta\& K. Acharya }\end{array}$ & $\begin{array}{l}\text { On the base } \\
\text { of Pterigotaalata (Stercaliace } \\
\text { ae) }\end{array}$ & $\begin{array}{l}\text { West Bengal, } \\
\text { (Botanical Garden } \\
\text { of the Ballygunge } \\
\text { Science College } \\
\text { campus, Kolkata) }\end{array}$ & $\begin{array}{l}\text { in Crous et } \\
\text { al., (2017) }\end{array}$ \\
\hline 17. & $\begin{array}{l}\text { Russula atropurpurea } \\
\text { (Krombh.) Britzelm. }\end{array}$ & $\begin{array}{l}\text { In leaf litter and } \\
\text { ectomycorrhizal } \\
\text { with Vateriaindica }\end{array}$ & $\begin{array}{l}\text { Chakrata, } \\
\text { Uttarakhand; } \\
\text { Malappuram and } \\
\text { Nilambur, Kerala } \\
\text { and Mangalore, } \\
\text { Karnataka }\end{array}$ & $\begin{array}{l}\text { Atri, Saini } \\
\text { 1986; Saini, } \\
\text { Atri (1989b); } \\
\text { Pavithra et } \\
\text { al., (2017); } \\
\text { Mohanan } \\
\text { (2011, 2014) }\end{array}$ \\
\hline 18. & $\begin{array}{l}\text { Russula atropurpurea } \\
\text { Peck. }\end{array}$ & $\begin{array}{l}\text { From conifer dominated } \\
\text { forest under pine }\end{array}$ & $\begin{array}{l}\text { Gulmarg, Jammu } \\
\text { and Kashmir }\end{array}$ & $\begin{array}{l}\text { Dar et al., } \\
(2010)\end{array}$ \\
\hline 19. & Russula aurataFr. & - & $\begin{array}{l}\text { Deoban, Chakrata, } \\
\text { Uttarakhand }\end{array}$ & $\begin{array}{l}\text { Atri and } \\
\text { Saini, (1986); } \\
\text { Saini and } \\
\text { Atri(1989b); } \\
\text { Saini } \text { et al., } \\
\text { (1989) }\end{array}$ \\
\hline
\end{tabular}




\begin{tabular}{|c|c|c|c|c|}
\hline 20. & Russula aurea Pers. & $\begin{array}{l}\text { From conifer dominated } \\
\text { forest }\end{array}$ & $\begin{array}{l}\text { Gulmarg, Jammu } \\
\text { and Kashmir }\end{array}$ & $\begin{array}{l}\text { Dar et al., } \\
(2010)\end{array}$ \\
\hline 21. & $\begin{array}{l}\text { Russula aureorubra K. } \\
\text { Das, A. Ghosh, } \\
\text { Baghela\&Buyck }\end{array}$ & $\begin{array}{l}\text { In temperate broadleaf forest } \\
\text { associated with Lithocarpus }\end{array}$ & Sikkim & $\begin{array}{l}\text { in Das et al., } \\
\text { (2017) }\end{array}$ \\
\hline 22. & $\begin{array}{l}\text { Russula aurora } \\
\text { (Krombh.) Bres. }\end{array}$ & - & $\begin{array}{l}\text { Chakrata, } \\
\text { Mussoorie, } \\
\text { Uttarakhand }\end{array}$ & $\begin{array}{l}\text { Saini et al., } \\
\text { (1989) }\end{array}$ \\
\hline 23. & Russula azureaBres. & $\begin{array}{l}\text { Growing solitary on } \\
\text { humicolous soil under } \\
\text { Juniperusrecurva }\end{array}$ & $\begin{array}{l}\text { Churdhar, Himachal } \\
\text { Pradesh }\end{array}$ & $\begin{array}{l}\text { Saini et al., } \\
\text { (2010) }\end{array}$ \\
\hline 24. & $\begin{array}{l}\text { Russula brevipesPeck } \\
=\text { Russula brevipesvar. } \\
\text { acriorShaffer }\end{array}$ & In forest areas & $\begin{array}{l}\text { Jageshwar,Mayawat } \\
\text { i, Champawat, } \\
\text { Uttarakhand; } \\
\text { Nagaland } \\
\text { (Puliebzie, } \\
\text { Zakhama, Chungtia) } \\
\text { and Namcha forest } \\
\text { ranges and Jammu } \\
\text { and Kashmir }\end{array}$ & $\begin{array}{l}\text { Das and } \\
\text { Sharma } \\
\text { (2005b); } \\
\text { Kumar } \text { et al., } \\
\text { (2014); } \\
\text { Watling and } \\
\text { Gregory } \\
(1980)\end{array}$ \\
\hline 25. & $\begin{array}{l}\text { Russula brevipes var. } \\
\text { acrior Shaffer }\end{array}$ & - & $\begin{array}{l}\text { Lohaghat, } \\
\text { Mayawati, } \\
\text { Champawat; } \\
\text { Sandev, } \\
\text { Pithoragarh, } \\
\text { Uttarakhand }\end{array}$ & $\begin{array}{l}\text { Das and } \\
\text { Sharma } \\
(2005 b)\end{array}$ \\
\hline 26. & $\begin{array}{l}\text { Russula } \\
\text { brunneoviolaceaCrawsh } \\
\text { ay var. macrosporaM. } \\
\text { Kaur, NS Atri, S. } \\
\text { Sharma \& Y. Singh }\end{array}$ & $\begin{array}{l}\text { Growing solitary on } \\
\text { humicolous soil in } \\
\text { Abiespindrowforest }\end{array}$ & $\begin{array}{l}\text { Narkanda, Himachal } \\
\text { Pradesh }\end{array}$ & $\begin{array}{l}\text { Kaur et al., } \\
\text { (2011) }\end{array}$ \\
\hline 27. & $\begin{array}{l}\text { Russula buyckii K. } \\
\text { Acharya, S. Paloi\& A.K. } \\
\text { Dutta }\end{array}$ & $\begin{array}{l}\text { Growing under Castanopsis } \\
\text { sp., among mosses }\end{array}$ & $\begin{array}{l}\text { Darjeeling, West } \\
\text { Bengal }\end{array}$ & $\begin{array}{l}\text { Paloi et al., } \\
\text { (2016) }\end{array}$ \\
\hline 28. & $\begin{array}{l}\text { Russula } \\
\text { brunneoviolaceavarrube } \\
\text { ogriseaRomang, }\end{array}$ & $\begin{array}{l}\text { Growing solitary on } \\
\text { humicolous soil in a broad } \\
\text { leaved forest }\end{array}$ & $\begin{array}{l}\text { Hattu Peak, } \\
\text { Himachal Pradesh }\end{array}$ & $\begin{array}{l}\text { Kaur et al., } \\
\text { (2011) }\end{array}$ \\
\hline 29. & $\begin{array}{l}\text { Russula californiensis } \\
\text { Burl }\end{array}$ & $\begin{array}{l}\text { Growing on hard ground in } \\
\text { August }\end{array}$ & $\begin{array}{l}\text { Kasauli, Himachal } \\
\text { Pradesh }\end{array}$ & $\begin{array}{l}\text { Chaudhary, } \\
\text { Tripathy } \\
\text { (2016) }\end{array}$ \\
\hline 30. & Russula cinerellaPat. & - & Wayanad, Kerala & $\begin{array}{l}\text { Mohanan } \\
(2011,2014)\end{array}$ \\
\hline 31. & $\begin{array}{l}\text { Russula cinnabarina } \\
\text { Berk. }\end{array}$ & On clay bank & $\begin{array}{l}\text { Darjeeling, West } \\
\text { Bengal }\end{array}$ & $\begin{array}{l}\text { Berkeley } \\
(1851)\end{array}$ \\
\hline 32. & Russula claroflava & - & Amravati, & Hedawoo \\
\hline
\end{tabular}




\begin{tabular}{|c|c|c|c|c|}
\hline & Grove & & Maharashtra & (2010) \\
\hline 33. & Russula compactaFrost & - & $\begin{array}{l}\text { Abbot Mt., Sandev, } \\
\text { DafiaDhura, } \\
\text { Uttarakhand }\end{array}$ & $\begin{array}{l}\text { Das and } \\
\text { Sharma } \\
(2005 b)\end{array}$ \\
\hline 34. & Russula congoanaPat. & - & $\begin{array}{l}\text { Mussoorie, } \\
\text { Uttarakhand; } \\
\text { Ernakulam, Kollam, } \\
\text { Malappuram, } \\
\text { Wayanad and } \\
\text { Thiruvananthapura } \\
\text { m, Kerala }\end{array}$ & $\begin{array}{l}\text { Sarwal } \\
(1984) ; \\
\text { Pradeep and } \\
\text { Vrinda } \\
\text { (2010); } \\
\text { Varghese et } \\
\text { al., (2010); } \\
\text { Mohanan } \\
\text { (2011) }\end{array}$ \\
\hline 35. & $\begin{array}{l}\text { Russula consobrina (Fr.) } \\
\text { Fr. }\end{array}$ & - & $\begin{array}{l}\text { Jamnotri, } \\
\text { Hanumanchatti, } \\
\text { Uttarakhand }\end{array}$ & $\begin{array}{l}\text { Atri and } \\
\text { Saini (1986, } \\
\text { 1990a) }\end{array}$ \\
\hline 36. & $\begin{array}{l}\text { Russula } \\
\text { cremeoavellanea Singer }\end{array}$ & - & $\begin{array}{l}\text { North West } \\
\text { Himalayas, } \\
\text { Himachal Pradesh }\end{array}$ & $\begin{array}{l}\text { Sharma et al., } \\
\text { (2016) }\end{array}$ \\
\hline 37. & Russula crustosa Peck. & $\begin{array}{l}\text { Solitary-scattered } \\
\text { underPiseasmithiana, } \\
\text { Pinuswalitiana, } \\
\text { Quercusincana and } \\
\text { Rhododendron arboretum }\end{array}$ & $\begin{array}{l}\text { Shimla, Himachal } \\
\text { Pradesh }\end{array}$ & $\begin{array}{l}\text { Bhatt and } \\
\text { Lakhanpal } \\
\text { (1988a) }\end{array}$ \\
\hline 38. & $\begin{array}{l}\text { Russula cyanoxantha } \\
\text { (Schaeff.) Fr. }\end{array}$ & Ectomycorrhizal on sal & $\begin{array}{l}\text { Gidhanisal forest, } \\
\text { West Bengal; } \\
\text { Chakrata, Kailana; } \\
\text { Joshimath, Oli } \\
\text { Forest; Ramgarh; } \\
\text { Lohaghat, } \\
\text { Champawat; } \\
\text { DafiaDhura, Maitly, } \\
\text { Uttarakhand }\end{array}$ & $\begin{array}{l}\text { Shajahan and } \\
\text { Samajpati } \\
\text { (1995); Atri } \\
\text { and Saini } \\
\text { (1986, } \\
\text { 1990c); Das } \\
\text { and Sharma } \\
\text { (2005b). }\end{array}$ \\
\hline 39. & $\begin{array}{l}\text { Russula dafianusK. Das } \\
\text { \& J.R. Sharma }\end{array}$ & - & $\begin{array}{l}\text { Dhakuri, } \\
\text { Bageshwar; } \\
\text { DafiaDhura; } \\
\text { Pithoragarh, } \\
\text { Uttarakhand }\end{array}$ & $\begin{array}{l}\text { Das and } \\
\text { Sharma } \\
(2005 b)\end{array}$ \\
\hline 40. & $\begin{array}{l}\text { Russula decipiens } \\
\text { (Singer) Bon }\end{array}$ & - & $\begin{array}{l}\text { Mukteshwar, } \\
\text { Nainital, } \\
\text { Uttarakhand }\end{array}$ & $\begin{array}{l}\text { Das and } \\
\text { Sharma } \\
(2005 b)\end{array}$ \\
\hline 41. & $\begin{array}{l}\text { Russula decolorans (Fr.) } \\
\text { Fr. }\end{array}$ & - & $\begin{array}{l}\text { Kumaon and } \\
\text { Champawat, } \\
\text { Mayawati; } \\
\text { Mornoulla, Almora; } \\
\text { Nainital, Ramgarh; }\end{array}$ & $\begin{array}{l}\text { Das and } \\
\text { Sharma } \\
\text { (2003) }\end{array}$ \\
\hline
\end{tabular}




\begin{tabular}{|c|c|c|c|c|}
\hline & & & Uttarakhand & \\
\hline$\overline{42 .}$ & Russula delica Fr. & Ectomycorrhizal on sal & $\begin{array}{l}\text { Gidhani and } \\
\text { Jhargram, West } \\
\text { Bengal and } \\
\text { Chakrata, Deoban } \\
\text { and } \\
\text { NainapeakUttarakha } \\
\text { nd }\end{array}$ & $\begin{array}{l}\text { Shajahan and } \\
\text { Samajpati } \\
\text { (1995); } \\
\text { Atri and } \\
\text { Saini (1986) }\end{array}$ \\
\hline 43. & $\begin{array}{l}\text { Russula } \\
\text { deliculaRomagn. }\end{array}$ & - & $\begin{array}{l}\text { Thiruvananthapura } \\
\text { m and Wayanad, } \\
\text { Kerala }\end{array}$ & $\begin{array}{l}\text { Pradeep and } \\
\text { Vrinda } \\
\text { (2010); } \\
\text { Mohanan } \\
(2011,2014)\end{array}$ \\
\hline$\overline{44 .}$ & $\begin{array}{l}\text { Russula densifoliaSecr. } \\
\text { ex Gillet }\end{array}$ & - & Jammu and Kashmir & $\begin{array}{l}\text { Watling and } \\
\text { Gregory } \\
\text { (1980) }\end{array}$ \\
\hline$\overline{45 .}$ & $\begin{array}{l}\text { Russula dhakurianaK. } \\
\text { Das, J.R. Sharma \& S.L. } \\
\text { Mills }\end{array}$ & $\begin{array}{l}\text { Associated with } \\
\text { Rhododendron }\end{array}$ & $\begin{array}{l}\text { Dhakuri, } \\
\text { Bageshwar, } \\
\text { Uttarakhand, } \\
\text { Himalaya }\end{array}$ & $\begin{array}{l}\text { Das et al., } \\
\text { (2006a) }\end{array}$ \\
\hline 46. & $\begin{array}{l}\text { Russula } \\
\text { dissimulansShaffer }\end{array}$ & - & $\begin{array}{l}\text { Adwani Forest, } \\
\text { Uttarakhand }\end{array}$ & $\begin{array}{l}\text { Bhatt et al., } \\
\text { (1995) }\end{array}$ \\
\hline$\overline{47 .}$ & $\begin{array}{l}\text { Russula dubdiana K. } \\
\text { Das, Atri\&Buyck }\end{array}$ & $\begin{array}{l}\text { On ground under } \\
\text { Castanopsishystrix in } \\
\text { subtropical to temperate } \\
\text { broad-leaved forest }\end{array}$ & Sikkim & $\begin{array}{l}\text { Das et al., } \\
\text { (2013) }\end{array}$ \\
\hline 48. & $\begin{array}{l}\text { Russula emetica } \\
\text { (Schaeff.) Pers. } \\
\text { 三AgaricusemeticusSchae } \\
\text { ff. }\end{array}$ & - & $\begin{array}{l}\text { Khasi Hills, } \\
\text { Meghalaya and clay } \\
\text { banks, Darjeeling, } \\
\text { West Bengal }\end{array}$ & $\begin{array}{l}\text { Berkeley } \\
(1856)\end{array}$ \\
\hline 49. & Russula farinipesRomell & $\begin{array}{l}\text { Growing in the mixed forest } \\
\text { dominated by Pinus } \\
\text { wallichiana and Quercus } \\
\text { incana }\end{array}$ & Himachal Predesh & $\begin{array}{l}\text { Saini and } \\
\text { Atri (1989) }\end{array}$ \\
\hline 50. & $\begin{array}{l}\text { Russula firmula Jul. } \\
\text { Schäff. }\end{array}$ & - & Jammu and Kashmir & $\begin{array}{l}\text { Watling, } \\
\text { Gregory } \\
\text { (1980) }\end{array}$ \\
\hline 51. & $\begin{array}{l}\text { Russula flavidavar. } \\
\text { Dhakurianus K. Das, } \\
\text { J.R. Sharma \& R.P. } \\
\text { Bhatt }\end{array}$ & - & $\begin{array}{l}\text { Dhakuri, } \\
\text { Bageshwar, } \\
\text { Uttarakhand }\end{array}$ & $\begin{array}{l}\text { Das and } \\
\text { Sharma } \\
\text { (2005a) }\end{array}$ \\
\hline 52. & Russula flocculosaBurl. & - & $\begin{array}{l}\text { Chobattakhal, } \\
\text { Phaedkhal, } \\
\text { PauriGarhwal, } \\
\text { Uttarakhand }\end{array}$ & $\begin{array}{l}\text { Bhatt et al., } \\
\text { (2007) }\end{array}$ \\
\hline
\end{tabular}




\begin{tabular}{|c|c|c|c|c|}
\hline 53. & Russula foetens Pers. & $\begin{array}{l}\text { On humicolous soil, under } \\
\text { Quercusincana, }\end{array}$ & $\begin{array}{l}\text { Joshimath, Nainital, } \\
\text { Chakrata, Deoban, } \\
\text { Jamnotri, } \\
\text { Uttarakhand; } \\
\text { Summer Hills, } \\
\text { Simla, Himachal } \\
\text { Pradesh }\end{array}$ & $\begin{array}{l}\text { Saini and } \\
\text { Atri (1984); } \\
\text { Saini and } \\
\text { Atri (1981) }\end{array}$ \\
\hline 54. & $\begin{array}{l}\text { Russula } \\
\text { fragrantissimaRomagn }\end{array}$ & - & $\begin{array}{l}\text { Nongkham, Namcha } \\
\text { and Tigit forest } \\
\text { range, Nagaland and } \\
\text { Jammu and Kashmir }\end{array}$ & $\begin{array}{l}\text { Kumar et al., } \\
\text { (2014); } \\
\text { Watling and } \\
\text { Gregory } \\
(1980)\end{array}$ \\
\hline 55. & Russula furcataPers. & Clay banks & $\begin{array}{l}\text { Sinchal, Sikkim, } \\
\text { Himalayas }\end{array}$ & $\begin{array}{l}\text { Berkeley } \\
(1856)\end{array}$ \\
\hline 56. & $\begin{array}{l}\text { Russula } \\
\text { grataBritzelm.=Russula } \\
\text { laurocerasiMelzer }\end{array}$ & $\begin{array}{l}\text { Growing solitary on } \\
\text { humicolous soil under } \\
\text { Quercusincana }\end{array}$ & $\begin{array}{l}\text { Thiruvananthapura } \\
\text { m, Kerala and } \\
\text { Himachal Pradesh }\end{array}$ & $\begin{array}{l}\text { Pradeep and } \\
\text { Vrinda } \\
(2010) ; \\
\text { Saini and } \\
\text { Atri (1989) }\end{array}$ \\
\hline 57. & $\begin{array}{l}\text { Russula griseocarnosa } \\
\text { X.H. Wang, Zhu L. } \\
\text { Yang \& Knudsen }\end{array}$ & - & Sikkim & $\begin{array}{l}\text { in Das et al., } \\
\text { (2010) }\end{array}$ \\
\hline 58. & Russula grossa Berk. & on earth and mossy bank & $\begin{array}{l}\text { Darjeeling, West } \\
\text { Bengal }\end{array}$ & $\begin{array}{l}\text { Berkeley } \\
\text { (1851) }\end{array}$ \\
\hline 59. & $\begin{array}{l}\text { Russula } \\
\text { himalayanaRawal\&Sarw } \\
\text { al }\end{array}$ & $\begin{array}{l}\text { On humus under Aesculus } \\
\text { and Acer }\end{array}$ & $\begin{array}{l}\text { JabbarKhet, } \\
\text { Mussoorie, } \\
\text { Uttarakhand }\end{array}$ & $\begin{array}{l}\text { Rawla and } \\
\text { Sarwal } \\
\text { (1983) }\end{array}$ \\
\hline 60. & $\begin{array}{l}\text { Russula hookeri S. Paloi, } \\
\text { A.K. Dutta\& K. Acharya }\end{array}$ & $\begin{array}{l}\text { Growing solitary - group of } \\
\text { two, under Castanopsis sp. } \\
\text { among the mosses }\end{array}$ & $\begin{array}{l}\text { Darjeeling, West } \\
\text { Bengal }\end{array}$ & $\begin{array}{l}\text { Paloi et al., } \\
\text { (2015) }\end{array}$ \\
\hline 61. & $\begin{array}{l}\text { Russula } \\
\text { hygrophyticaPegler }\end{array}$ & - & $\begin{array}{l}\text { Ernakulam, } \\
\text { Perumbavoorand } \\
\text { Thiruvananthapura } \\
\text { m, Kerala }\end{array}$ & $\begin{array}{l}\text { Mohanan } \\
(2011,2014)\end{array}$ \\
\hline 62. & $\begin{array}{l}\text { Russula indicaSathe\& } \\
\text { J.T. Daniel }\end{array}$ & 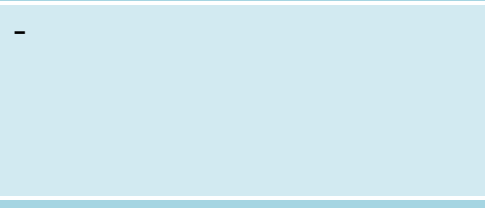 & Idukki, Kerala & $\begin{array}{l}\text { in Sathe } e t \\
\text { al., (1980); } \\
\text { Florence } \\
\text { (2004) }\end{array}$ \\
\hline 63. & $\begin{array}{l}\text { Russula indoarmeniaca } \\
\text { A. Ghosh, K. Das \& R.P. } \\
\text { Bhatt }\end{array}$ & From broadleaf forest & $\begin{array}{l}\text { Baniyakund, } \\
\text { Rudraprayag, } \\
\text { Uttarakhand }\end{array}$ & $\begin{array}{l}\text { Ghosh et al., } \\
\text { (2016) }\end{array}$ \\
\hline 64. & $\begin{array}{l}\text { Russula } \\
\text { intervenosaPaloi, A.K. } \\
\text { Dutta\& K. Acharya }\end{array}$ & $\begin{array}{l}\text { Associated with } \\
\text { Shorearobusta }\end{array}$ & $\begin{array}{l}\text { Lodhasuli forest, } \\
\text { PaschimMidnapur, } \\
\text { West Bengal }\end{array}$ & $\begin{array}{l}\text { in Crous et } \\
\text { al., (2016) }\end{array}$ \\
\hline 65. & Russula kanadii A.K. & Growing solitary under & Gurguripal forest, & Dutta et al., \\
\hline
\end{tabular}




\begin{tabular}{|c|c|c|c|c|}
\hline & Dutta\& K. Acharya & Shorearobusta tree & $\begin{array}{l}\text { West Midnapur } \\
\text { District, West } \\
\text { Bengal }\end{array}$ & (2015) \\
\hline 66. & $\begin{array}{l}\text { Russula khanchanjungae } \\
\text { Van de Putte, K. Das } \\
\text { \&Buyck (as } \\
\text { 'khanchanjungae) }\end{array}$ & $\begin{array}{l}\text { in mixed forest with } \\
\text { Abiesdensa, Betulautilis and } \\
\text { Rhododendronbarbatum }\end{array}$ & Sikkim & $\begin{array}{l}\text { in Das et al., } \\
\text { (2010) }\end{array}$ \\
\hline 67. & $\begin{array}{l}\text { Russula leelavathyi K.B. } \\
\text { Vrinda, C.K. Pradeep\& } \\
\text { T.K. Abraham }\end{array}$ & $\begin{array}{l}\text { On sandy soil, associated } \\
\text { with Hopeaparviflora }\end{array}$ & $\begin{array}{l}\text { Thiruvananthapura } \\
\text { m, Malappuram and } \\
\text { Wayanad, Kerala } \\
\text { Madhya Pradesh }\end{array}$ & $\begin{array}{l}\text { Vrinda et al., } \\
\text { (1997a); } \\
\text { Pradeep and } \\
\text { Vrinda } \\
\text { (2007, 2010); } \\
\text { Mohanan } \\
(2011,2014)\end{array}$ \\
\hline 68. & Russula lepidaFr. & $\begin{array}{l}\text { ectomycorrhizal on sal; on } \\
\text { clay bank, Darjeeling, WB; } \\
\text { reported edible from } \\
\text { Tapovan, Dehradun, } \\
\text { Uttrakhand }\end{array}$ & $\begin{array}{l}\text { Gidhani and } \\
\text { Jhargramsal forest, } \\
\text { West Bengal; } \\
\text { Uttrakhand }\end{array}$ & $\begin{array}{l}\text { Shajahan and } \\
\text { Samajpati } \\
\text { (1995); } \\
\text { (Berkeley } \\
\text { (1851); } \\
\text { Semwal } \text { et } \\
\text { al., (2014) }\end{array}$ \\
\hline 69. & $\begin{array}{l}\text { Russula lepidicolor } \\
\text { Romagnesi }\end{array}$ & $\begin{array}{l}\text { growing scattered on } \\
\text { humicolous soil in } \\
\text { Cedrusdeodara forest }\end{array}$ & $\begin{array}{l}\text { Nagar Forest, } \\
\text { Manali, Himachal } \\
\text { Pradesh }\end{array}$ & $\begin{array}{l}\text { Saini et al., } \\
\text { (2010) }\end{array}$ \\
\hline 70. & $\begin{array}{l}\text { Russula lutea, Bhatt et } \\
\text { al., 1988a, b }\end{array}$ & $\begin{array}{l}\text { Solitary-scattered, associated } \\
\text { with Cedrusdeodara, } \\
\text { Piceasmithiana, } \\
\text { Pinuswalitiana, } \\
\text { Quercusincana and } \\
\text { Rhododendron arboreum and } \\
\text { humicolous soil in sal forests }\end{array}$ & $\begin{array}{l}\text { Shimla, Himachal } \\
\text { Pradesh and lower } \\
\text { Shiwalik Hills of } \\
\text { Uttarakhand }\end{array}$ & $\begin{array}{l}\text { Bhatt and } \\
\text { Lakhanpal } \\
\text { (1988b); } \\
\text { Semwal et } \\
\text { al., (2014I }\end{array}$ \\
\hline 71. & Russula luteotacta Rea & - & $\begin{array}{l}\text { Thiruvananthapura } \\
\text { m, Ernakulam and } \\
\text { Perumbavoor, } \\
\text { Kerala }\end{array}$ & $\begin{array}{l}\text { Pradeep and } \\
\text { Vrinda } \\
\text { (2010); } \\
\text { Mohanan } \\
(2011,2014)\end{array}$ \\
\hline 72. & Russula mariae Peck & $\begin{array}{l}\text { On soil under Hopeaponga, } \\
\text { H. parviflora, } \\
\text { Vateriaindica,Diospyrosmala } \\
\text { barica forming } \\
\text { ectomycorrhiza }\end{array}$ & $\begin{array}{l}\text { Shenkily, } \\
\text { Kulathupuzha, } \\
\text { Wayanad, Kerala }\end{array}$ & $\begin{array}{l}\text { Mohanan } \\
(2014)\end{array}$ \\
\hline 73. & Russula martinica Pegler & $\begin{array}{l}\text { On soil } \\
\text { underHopeaparviflora }\end{array}$ & $\begin{array}{l}\text { Malappuram, } \\
\text { Nilambur, Kerala }\end{array}$ & $\begin{array}{l}\text { Mohanan } \\
(2011,2014)\end{array}$ \\
\hline 74. & $\begin{array}{l}\text { Russula mayawatianaK. } \\
\text { Das, S.L. Mill. \& J.R. } \\
\text { Sharma }\end{array}$ & $\begin{array}{l}\text { Associated with Quercus and } \\
\text { Rhododendron }\end{array}$ & $\begin{array}{l}\text { Champawat, } \\
\text { Mayawati, } \\
\text { Uttarakhand }\end{array}$ & $\begin{array}{l}\text { Das K et al., } \\
\text { (2006a) }\end{array}$ \\
\hline
\end{tabular}




\begin{tabular}{|c|c|c|c|c|}
\hline 75. & $\begin{array}{l}\text { Russula michiganensis } \\
\text { Shaffer }\end{array}$ & $\begin{array}{l}\text { Under Hopeaponga, } H . \\
\text { parviflora, Vateriaindicaand } \\
\text { Diospyrosmalabarica }\end{array}$ & $\begin{array}{l}\text { from Wayanad and } \\
\text { Ernakulam, Kerala }\end{array}$ & $\begin{array}{l}\text { Mohanan } \\
(2011,2014)\end{array}$ \\
\hline 76. & $\begin{array}{l}\text { Russula minutulavar. } \\
\text { Minutula Velen. }\end{array}$ & - & $\begin{array}{l}\text { Kailana, Dhobighat, } \\
\text { Alumandi, } \\
\text { Mussoorie, } \\
\text { Joshimath, } \\
\text { Uttarakhand }\end{array}$ & $\begin{array}{l}\text { Saini and } \\
\text { Atri (1984); } \\
\text { Saini } \text { et al., } \\
\text { (1989) }\end{array}$ \\
\hline 77. & $\begin{array}{l}\text { Russula } \\
\text { mukteshwaricaK. Das, } \\
\text { S.L. Mill., J.R. Sharma } \\
\text { \& R.P. Bhatt }\end{array}$ & $\begin{array}{l}\text { In close association with } \\
\text { Myrica, Quercus and } \\
\text { Rhododendron }\end{array}$ & $\begin{array}{l}\text { Uttarakhand, } \\
\text { Himalayas }\end{array}$ & $\begin{array}{l}\text { Das K et al., } \\
(2005)\end{array}$ \\
\hline 78. & $\begin{array}{l}\text { Russula } \\
\text { mussooriensisRawla\&Sa } \\
\text { rwal }\end{array}$ & - & $\begin{array}{l}\text { from JabbarKhet, } \\
\text { Mussoorie, } \\
\text { Uttarakhand }\end{array}$ & $\begin{array}{l}\text { Rawla and } \\
\text { Sarwal } \\
(1983)\end{array}$ \\
\hline 79. & $\begin{array}{l}\text { Russula mustelinaFr., } \\
\text { Oli Forest }\end{array}$ & - & $\begin{array}{l}\text { Chakrata, Kailana, } \\
\text { Uttarakhand }\end{array}$ & $\begin{array}{l}\text { Saini and } \\
\text { Atri (1984); } \\
\text { (Atri and } \\
\text { Saini (1986, } \\
\text { 1990c) }\end{array}$ \\
\hline 80. & $\begin{array}{l}\text { Russula natarjaniiK. } \\
\text { Das, J.R. Sharma \&Atri }\end{array}$ & $\begin{array}{l}\text { Associated with } \\
\text { Quercus species in moist, } \\
\text { temperate, deciduous/mixed } \\
\text { forests }\end{array}$ & $\begin{array}{l}\text { Dhakuri, } \\
\text { Bageshwar, } \\
\text { Uttarakhand }\end{array}$ & $\begin{array}{l}\text { Das et al., } \\
(2006 \mathrm{~b})\end{array}$ \\
\hline 81. & $\begin{array}{l}\text { Russula nauseosa (Pers.) } \\
\text { Fr. }\end{array}$ & $\begin{array}{l}\text { On ground amongst pine } \\
\text { litter }\end{array}$ & $\begin{array}{l}\text { Gulmatg, Jammu } \\
\text { and Kashmir }\end{array}$ & $\begin{array}{l}\text { Abraham et } \\
\text { al., (1980); } \\
\text { Watling and } \\
\text { Gregory } \\
(1980)\end{array}$ \\
\hline 82. & Russula nigricansFr. & - & $\begin{array}{l}\text { Chakrata, } \\
\text { Uttarakhand }\end{array}$ & $\begin{array}{l}\text { Saini and } \\
\text { Atri (1984); } \\
\text { Saini } \text { et al., } \\
\text { (1988) }\end{array}$ \\
\hline 83. & Russula nobilis Velen & In forest & $\begin{array}{l}\text { Pherma and } \\
\text { Mankoi, Nagaland }\end{array}$ & $\begin{array}{l}\text { Kumar et al., } \\
\text { (2014) }\end{array}$ \\
\hline 84. & $\begin{array}{l}\text { Russula obscuricolor K. } \\
\text { Das, A. Ghosh\&Buyck }\end{array}$ & $\begin{array}{l}\text { On soil in mixed broad leaf } \\
\text { forest associated with } \\
\text { Castanopsis }\end{array}$ & Sikkim & $\begin{array}{l}\text { (in Das et al., } \\
\text { (2017) }\end{array}$ \\
\hline 85. & $\begin{array}{l}\text { Russula ochroleuca } \\
\text { (Pers.) FrGray. }\end{array}$ & In forest ranges & $\begin{array}{l}\text { Lahorijan, } \\
\text { Puliebzie, Zakhama, } \\
\text { Nagaland }\end{array}$ & $\begin{array}{l}\text { Kumar et al., } \\
\text { (2014) }\end{array}$ \\
\hline 86. & $\begin{array}{l}\text { Russula } \\
\text { ochroleuca(Pers.) Fr. }\end{array}$ & - & $\begin{array}{l}\text { Chakrata, } \\
\text { Uttarakhand }\end{array}$ & $\begin{array}{l}\text { Saini et al., } \\
\text { (1989) }\end{array}$ \\
\hline 87. & $\begin{array}{l}\text { Russula } \\
\text { odorataRomagn. }\end{array}$ & - & $\begin{array}{l}\text { JabbarKhet, } \\
\text { Mussoorie, }\end{array}$ & $\begin{array}{l}\text { Rawla and } \\
\text { Sarwal }\end{array}$ \\
\hline
\end{tabular}




\begin{tabular}{|c|c|c|c|c|}
\hline & & & Uttarakhand & (1983) \\
\hline 88. & $\begin{array}{l}\text { Russula } \\
\text { olivacea(Schaeff.) Fr. }\end{array}$ & $\begin{array}{l}\text { In dense clusters, around } \\
\text { trunks, in pine forest }\end{array}$ & $\begin{array}{l}\text { Shyrwat, Shillong, } \\
\text { Meghalaya }\end{array}$ & $\begin{array}{l}\text { Kalita et al., } \\
\text { (2016) }\end{array}$ \\
\hline 89. & $\begin{array}{l}\text { Russula parazurea Jul. } \\
\text { Schäff. }\end{array}$ & - & $\begin{array}{l}\text { Chennai, Tamil } \\
\text { Nadu }\end{array}$ & $\begin{array}{l}\text { Natarajan and } \\
\text { Raman } \\
(1983 c)\end{array}$ \\
\hline 90. & $\begin{array}{l}\text { Russula } \\
\text { parvovirescensBuyck, D. } \\
\text { Mitch. \&Parrent }\end{array}$ & $\begin{array}{l}\text { Solitary, on ground, in Pine } \\
\text { forest }\end{array}$ & $\begin{array}{l}\text { Shyrwat, Shillong, } \\
\text { Meghalaya }\end{array}$ & $\begin{array}{l}\text { Kalita et al., } \\
(2016)\end{array}$ \\
\hline 91. & $\begin{array}{l}\text { Russula pauriensis A. } \\
\text { Ghosh, K. Das \&Buyck }\end{array}$ & $\begin{array}{l}\text { On soil in mixed broadleaf } \\
\text { forest associated with } \\
\text { Quercus }\end{array}$ & Uttarakhand & $\begin{array}{l}\text { in Das et al., } \\
(2017)\end{array}$ \\
\hline 92. & Russula peckii Singer & - & $\begin{array}{l}\text { from North West } \\
\text { Himalayas, } \\
\text { Himachal Pradesh }\end{array}$ & $\begin{array}{l}\text { Sharma et al., } \\
\text { (2016) }\end{array}$ \\
\hline 93. & $\begin{array}{l}\text { Russula pectinata(Bull.) } \\
\text { Fr. }\end{array}$ & - & $\begin{array}{l}\text { JabbarKhet, } \\
\text { Mussoorie, } \\
\text { Uttarakhand }\end{array}$ & $\begin{array}{l}\text { Rawla and } \\
\text { Sarwal } \\
(1983)\end{array}$ \\
\hline 94. & Russula pectinataFr. & $\begin{array}{l}\text { On humicolous soil under } \\
\text { Quercusincana, mycorrhizal } \\
\text { with sal }\end{array}$ & $\begin{array}{l}\text { Chakrata, } \\
\text { Uttarakhand and } \\
\text { Gidhanisal forest, } \\
\text { West Bengal }\end{array}$ & $\begin{array}{l}\text { (Saini SS, } \\
\text { Atri NS, } \\
\text { 1989); } \\
\text { (Shajahan M } \\
\text { and } \\
\text { Samajpati N, } \\
\text { 1995) }\end{array}$ \\
\hline 95. & $\begin{array}{l}\text { Russula periglypta } \text { Berk. } \\
\& \text { Broome }\end{array}$ & $\begin{array}{l}\text { On soil under Vateriaindica, } \\
\text { Hopeaparvifloraand } \\
\text { Diospyrosmalabarica }\end{array}$ & $\begin{array}{l}\text { Thiruvananthapura } \\
\text { m, Nilambur and } \\
\text { Malappuram, Kerala }\end{array}$ & $\begin{array}{l}\text { Pradeep and } \\
\text { Vrinda } \\
\text { (2010); } \\
\text { Manimohan, } \\
\text { Deepna } \\
\text { (2011); } \\
\text { Mohanan } \\
(2011,2014)\end{array}$ \\
\hline 96. & $\begin{array}{l}\text { Russula } \\
\text { persicinaKrombh. }\end{array}$ & - & Jammu and Kashmir & $\begin{array}{l}\text { Watling and } \\
\text { Gregory } \\
(1980)\end{array}$ \\
\hline 97. & $\begin{array}{l}\text { Russula petersenii A. } \\
\text { Ghosh\& K. Das }\end{array}$ & $\begin{array}{l}\text { On the soil under } \\
\text { Rhododendron }\end{array}$ & Uttarakhand & $\begin{array}{l}\text { Ghosh and } \\
\text { Das (2017) }\end{array}$ \\
\hline 98. & $\begin{array}{l}\text { Russula } \\
\text { praetervisaSarnari }\end{array}$ & - & $\begin{array}{l}\text { Dhakuri, } \\
\text { Bageshwar; } \\
\text { Mayawati and } \\
\text { Mornoula, } \\
\text { Champawat, } \\
\text { Uttarakhand }\end{array}$ & $\begin{array}{l}\text { Sharma et al., } \\
\text { (2005) }\end{array}$ \\
\hline 99. & Russula & - & Phedkhal, & Bhatt et al., \\
\hline
\end{tabular}




\begin{tabular}{|c|c|c|c|c|}
\hline & pseudolepidaSinger & & $\begin{array}{l}\text { PauriGarhwal; } \\
\text { Mandal, Chamoli, } \\
\text { Uttarakhand }\end{array}$ & (2007) \\
\hline 100. & $\begin{array}{l}\text { Russula } \\
\text { puellarisvarpuellaria Fr. }\end{array}$ & $\begin{array}{l}\text { Solitary on humicolous soil } \\
\text { among Fragaria sp., under } \\
\text { Cedrusdeodara }\end{array}$ & $\begin{array}{l}\text { Narkanda, Himachal } \\
\text { Pradesh }\end{array}$ & $\begin{array}{l}\text { Kaur et al., } \\
(2011)\end{array}$ \\
\hline 101. & $\begin{array}{l}\text { Russula puellarisvar. } \\
\text { atriiK. Das, S.L. Mill. \& } \\
\text { J. R Sharma }\end{array}$ & $\begin{array}{l}\text { Associated with Quercus and } \\
\text { Rhododendron }\end{array}$ & $\begin{array}{l}\text { Dhakuri, } \\
\text { Bageshwar, } \\
\text { Uttarakhand, } \\
\text { (Himalayas) }\end{array}$ & $\begin{array}{l}\text { Das et al., } \\
\text { (2006a) }\end{array}$ \\
\hline 102. & $\begin{array}{l}\text { Russula } \\
\text { pulverulentaPeck }\end{array}$ & - & $\begin{array}{l}\text { Teka, Gandera, } \\
\text { PauriGarhwal, } \\
\text { Uttarakhand }\end{array}$ & $\begin{array}{l}\text { Bhatt et al., } \\
\text { (2007) }\end{array}$ \\
\hline 103. & $\begin{array}{l}\text { Russula } \\
\text { purpureonigraPetch }\end{array}$ & - & $\begin{array}{l}\text { Thiruvananthapura } \\
\text { m and Malappuram, } \\
\text { Kerala }\end{array}$ & $\begin{array}{l}\text { Pradeep and } \\
\text { Vrinda } \\
(2010) \text {; } \\
\text { Manimohan } \\
\text { and Deepna } \\
\text { (2011) }\end{array}$ \\
\hline 104. & $\begin{array}{l}\text { Russula rajendraeA. } \\
\text { Ghosh\& K. Das }\end{array}$ & On the soil under Quercus & Uttarakhand & $\begin{array}{l}\text { Ghosh and } \\
\text { Das (2017) }\end{array}$ \\
\hline 105. & Russula raoultiiQuél. & - & $\begin{array}{l}\text { DafiaDhura, } \\
\text { Pithoragarh; } \\
\text { Mornoulla, Almora, } \\
\text { Uttarakhand }\end{array}$ & $\begin{array}{l}\text { Das and } \\
\text { Sharma } \\
(2003)\end{array}$ \\
\hline 106. & $\begin{array}{l}\text { Russula } \\
\text { rhodomelaneaSarnari }\end{array}$ & - & $\begin{array}{l}\text { Dhakuri, } \\
\text { Bageshwar, } \\
\text { Uttarakhand }\end{array}$ & $\begin{array}{l}\text { Das and } \\
\text { Sharma } \\
(2001 b)\end{array}$ \\
\hline 107. & $\begin{array}{l}\text { Russula romagnesiana } \\
\text { Schaffer }\end{array}$ & - & $\begin{array}{l}\text { Thiruvananthapura } \\
\text { m, Kerala }\end{array}$ & $\begin{array}{l}\text { Pradeep and } \\
\text { Vrinda } \\
(2010)\end{array}$ \\
\hline 108. & $\begin{array}{l}\text { Russula rubra(Lam.) } \\
\text { Fr.=Russula rubra var. } \\
\text { hymenocystidiataAtri\& } \\
\text { Kour }\end{array}$ & - & Punjab & $\begin{array}{l}\text { Atri and } \\
\text { Kour (2003) }\end{array}$ \\
\hline 109. & $\begin{array}{l}\text { Russula } \\
\text { sanguinaria(Schumach. } \\
\text { )Rauschert =Russula } \\
\text { rosacea (Pers.) Gray } \\
\text { =AgaricusroseusSchaeff } \\
\text {. }\end{array}$ & $\begin{array}{l}\text { In pine wood; on the } \\
\text { ground }\end{array}$ & $\begin{array}{l}\text { Sikkim, Himalayas } \\
\text { and } \\
\text { Mussoorie,Uttarak } \\
\text { hand }\end{array}$ & $\begin{array}{l}\text { Berkeley } \\
\text { (1851a); } \\
\text { Mundkur } \\
\text { (1938) }\end{array}$ \\
\hline 110. & $\begin{array}{l}\text { Russula sarnarii A. } \\
\text { Ghosh, K. Das \& R.P. } \\
\text { Bhatt }\end{array}$ & $\begin{array}{l}\text { Growing under Pinus } \\
\text { forest }\end{array}$ & Uttarakhand & $\begin{array}{l}\text { Ghosh et al., } \\
\text { (2017) }\end{array}$ \\
\hline 111. & Russula sanguinea $\mathrm{Fr}$. & - & from Jammu and & Watling and \\
\hline
\end{tabular}




\begin{tabular}{|c|c|c|c|c|}
\hline & $\begin{array}{l}=\text { =Agaricussanguineus B } \\
\text { ull. }\end{array}$ & & Kashmir & $\begin{array}{l}\text { Gregory } \\
(\mathbf{1 9 8 0 )}\end{array}$ \\
\hline 112 & $\begin{array}{l}\text { Russula } \\
\text { schaefferinaRawla\&Sar } \\
\text { wal }\end{array}$ & - & $\begin{array}{l}\text { Mussoorie, } \\
\text { Uttarakhand }\end{array}$ & $\begin{array}{l}\text { Rawla and } \\
\text { Sarwal } \\
\text { (1983) }\end{array}$ \\
\hline 113. & Russula senecis S. Imai & $\begin{array}{l}\text { In association with } \\
\text { Vateriaindica, dipterocarp } \\
\text { forests, and under } \\
\text { Lithocarpus and Castenopsis }\end{array}$ & $\begin{array}{l}\text { Western Ghats } \\
\text { Sikkim } \\
\text { West Bengal }\end{array}$ & $\begin{array}{l}\text { Natarajan et } \\
\text { al., (2005); } \\
\text { Das (2009); } \\
\text { Das et al., } \\
\text { (2010); } \\
\text { Khatua } \text { et al., } \\
\text { (2015) }\end{array}$ \\
\hline 114. & $\begin{array}{l}\text { Russula sharmaeK. Das, } \\
\text { Atri\&Buyck }\end{array}$ & $\begin{array}{l}\text { On ground under } \\
\text { Lithocarpuspachyphyllus in } \\
\text { mixed forest }\end{array}$ & Sikkim & $\begin{array}{l}\text { Das et al., } \\
(2013)\end{array}$ \\
\hline 115. & $\begin{array}{l}\text { Russula shingbaensis K. } \\
\text { Das \& S.L. Mill. }\end{array}$ & $\begin{array}{l}\text { On ground, sub-alpine forest, } \\
\text { under Abiesdensa, } \\
\text { ShingbaRhododendron } \\
\text { wildlife sanctuary }\end{array}$ & $\begin{array}{l}\text { North district, } \\
\text { Sikkim }\end{array}$ & $\begin{array}{l}\text { Das et al., } \\
\text { (2014). }\end{array}$ \\
\hline 116. & $\begin{array}{l}\text { Russula sikkimensis K. } \\
\text { Das, Atri\&Buyck }\end{array}$ & $\begin{array}{l}\text { On ground in Abiesdensa } \\
\text { sub-alpine mixed forest }\end{array}$ & Sikkim & $\begin{array}{l}\text { Das et al., } \\
\text { (2013) }\end{array}$ \\
\hline 117. & $\begin{array}{l}\text { Russula silvicolaShaffer, } \\
\text { Beih., Khirsu }\end{array}$ & - & $\begin{array}{l}\text { PauriGrahwal, } \\
\text { Uttarakhand }\end{array}$ & $\begin{array}{l}\text { Bhatt et al., } \\
\text { (2007) }\end{array}$ \\
\hline 118. & $\begin{array}{l}\text { Russula subfoetensW.G. } \\
\text { Sm. }\end{array}$ & $\begin{array}{l}\text { Solitary on humicolous soil } \\
\text { unerCedrusdeodaraand } \\
\text { Quercusincana }\end{array}$ & $\begin{array}{l}\text { Chakrata, } \\
\text { Uttarakhand }\end{array}$ & $\begin{array}{l}\text { Atri and } \\
\text { Saini (1986); } \\
\text { Saini and } \\
\text { Atri (1989a) }\end{array}$ \\
\hline 119. & $\begin{array}{l}\text { Russula thindii K. Das \& } \\
\text { S.L. Mill. }\end{array}$ & $\begin{array}{l}\text { On ground, coniferous forest, } \\
\text { Abiesdensa, Shingba } \\
\text { Rhododendron wildlife } \\
\text { sanctuary, }\end{array}$ & $\begin{array}{l}\text { North district, } \\
\text { Sikkim }\end{array}$ & $\begin{array}{l}\text { Das et al., } \\
(2014)\end{array}$ \\
\hline 120. & $\begin{array}{l}\text { Russula tsokae K. Das, } \\
\text { Van de Putte\&Buyck }\end{array}$ & Under Tsugadumosa forest & Sikkim & $\begin{array}{l}\text { in Das et al., } \\
\text { (2010) }\end{array}$ \\
\hline 121. & $\begin{array}{l}\text { Russula vaurasiana K. } \\
\text { Das \& J.R. Sharma }\end{array}$ & $\begin{array}{l}\text { Associated with Quercus in } \\
\text { temperate mixed forest }\end{array}$ & Uttarakhand & $\begin{array}{l}\text { Das and } \\
\text { Sharma } \\
(2005 b)\end{array}$ \\
\hline 122. & $\begin{array}{l}\text { Russula velenovskyi } \\
\text { Melzer\&Zvára }\end{array}$ & In Oli Forest & $\begin{array}{l}\text { Joshimath, } \\
\text { Uttarakhand }\end{array}$ & $\begin{array}{l}\text { Atri and } \\
\text { Saini (1986); } \\
\text { Saini and } \\
\text { Atri (1989b) }\end{array}$ \\
\hline 123. & Russula vescaFr. & Ectomycorrhizal on sal, & $\begin{array}{l}\text { Gidhani and } \\
\text { Balibhasasal forest, } \\
\text { West Bengal }\end{array}$ & $\begin{array}{l}\text { Shajahan and } \\
\text { Samajpati } \\
\text { (1995) }\end{array}$ \\
\hline 124. & $\begin{array}{l}\text { Russula } \\
\text { virescens(Schaeff.) Fr., }\end{array}$ & - & $\begin{array}{l}\text { Dhobighat, } \\
\text { Mussoorie; }\end{array}$ & $\begin{array}{l}\text { Saini et al., } \\
\text { (1988) }\end{array}$ \\
\hline
\end{tabular}




\section{Chakrata, Deoban,} Uttarakhand

Table.2 Distribution of 124 species of Russula in different states of India

\begin{tabular}{|l|l|c|c|}
\hline S. No. & State & Number of species & Percentage (\%) \\
\hline 1. & Himachal Pradesh & 13 & 9.2 \\
\hline 2. & Jammu and Kashmir & 10 & 7.1 \\
\hline 3. & Karnataka & 2 & 1.4 \\
\hline 4. & Kerala & 17 & 12.1 \\
\hline 5. & Madhya Pradesh & 6 & 4.3 \\
\hline 6. & Maharashtra & 1 & 0.7 \\
\hline 7. & Meghalaya & 3 & 2.1 \\
\hline 8. & Nagaland & 6 & 4.3 \\
\hline 9. & Punjab & 2 & 1.4 \\
\hline 10. & Sikkim & 10 & 7.1 \\
\hline 11. & Tamil Nadu & 1 & 0.7 \\
\hline 12. & Uttarakhand & 57 & 40.4 \\
\hline 13. & West Bengal & 13 & 9.2 \\
\hline & Toal & 141 & 100 \\
\hline
\end{tabular}

Table.3 Edible species of Russula

\begin{tabular}{|c|c|c|c|}
\hline S. No. & Name of species & Place & Reference \\
\hline 1. & Russula crustosa & Himachal Pradesh & Bhatt and Lakhanpal (1988a, b) \\
\hline 2. & Russula lepida & $\begin{array}{l}\text { Tapovan, Dehradun, } \\
\text { Uttarakhand }\end{array}$ & Semwal et al., (2014) \\
\hline 3. & Russula lutea & $\begin{array}{l}\text { Himachal Pradesh and } \\
\text { Uttarakhand }\end{array}$ & $\begin{array}{l}\text { Bhatt and Lakhanpal (1988b); } \\
\text { Semwal et al., (2014) }\end{array}$ \\
\hline 4. & Russula olivacea & Meghalaya & Kalita et al., (2016) \\
\hline 5. & Russula parvovirescens & Meghalaya & Kalita et al., (2016) \\
\hline 6. & Russula senecis & West Bengal & Khatua et al., (2015) \\
\hline 7. & Russula virescens & Uttarakhand & Saini et al., (1988) \\
\hline
\end{tabular}


Fig.1-4 Russula adusta, 1 basidiocarps, 2 basidia, 3 basisiospores (100x).

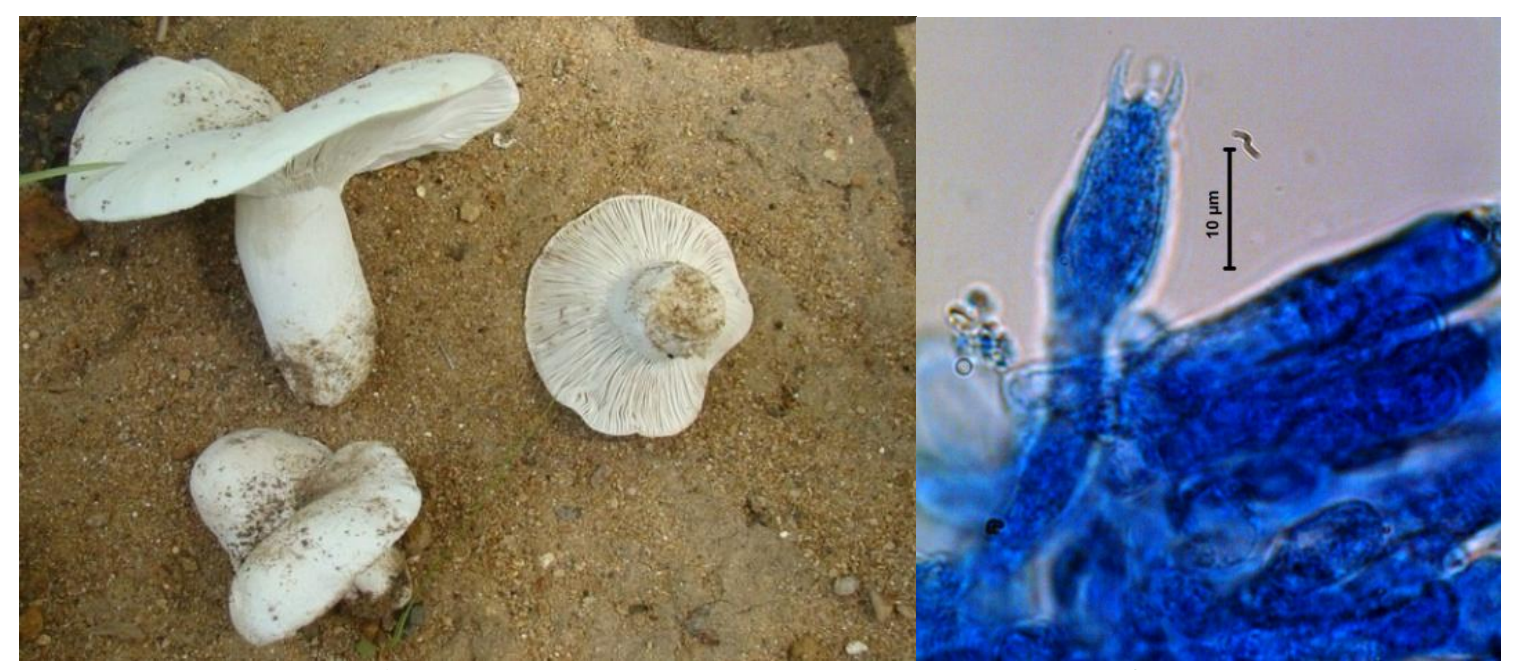

1

2

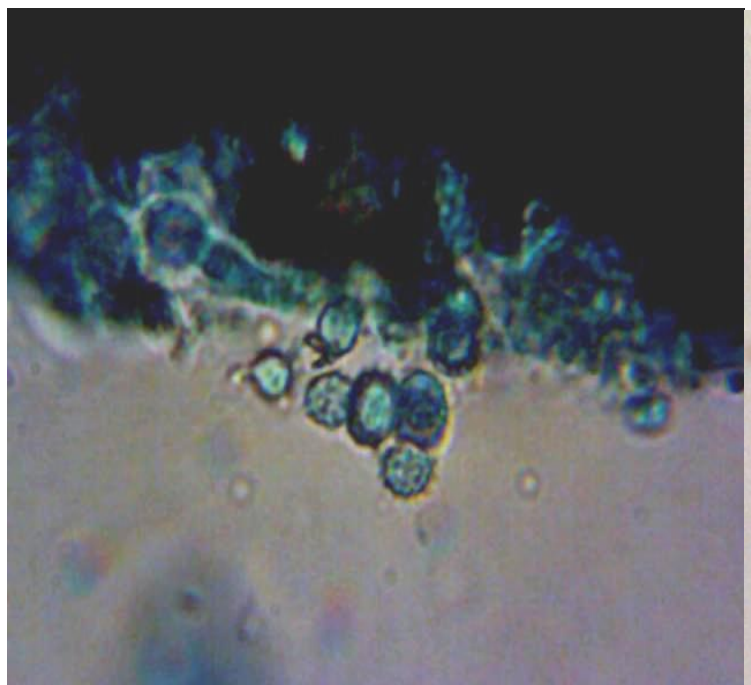

3
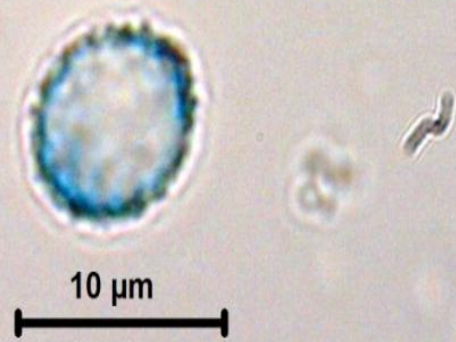

4

Fig.5-7 Russula cinerella, 4 basidiocarp, 6 basidia and 6 basidiospores

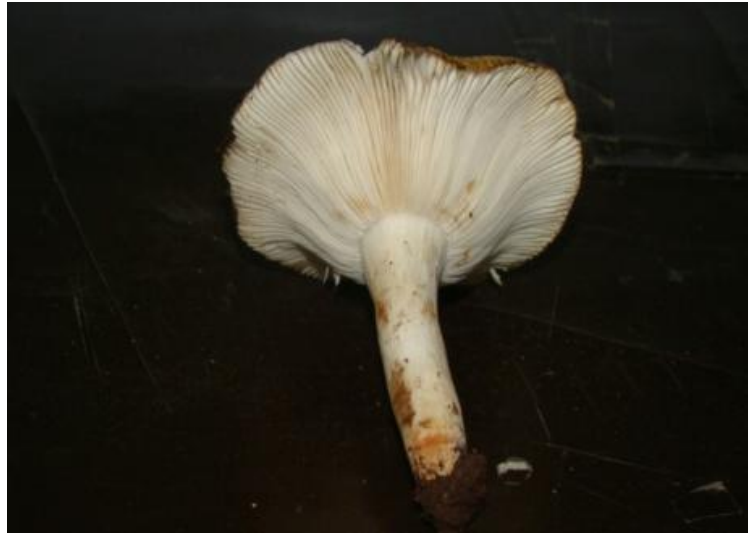

5

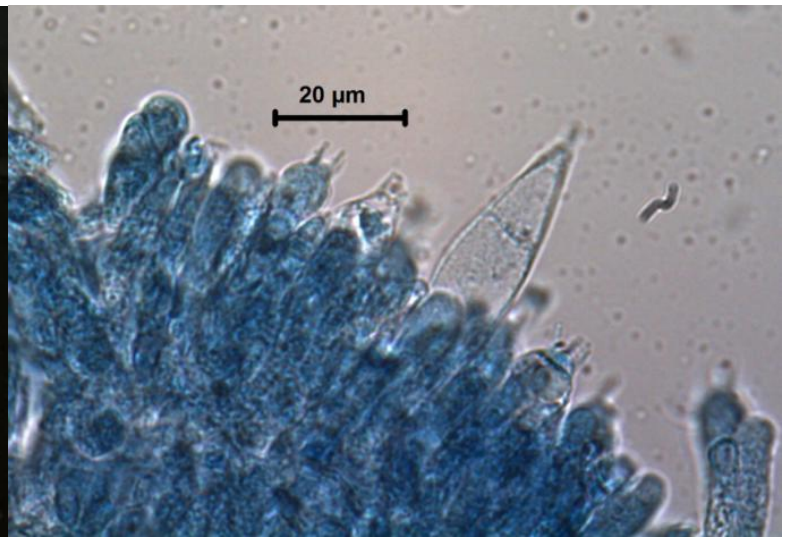

6 


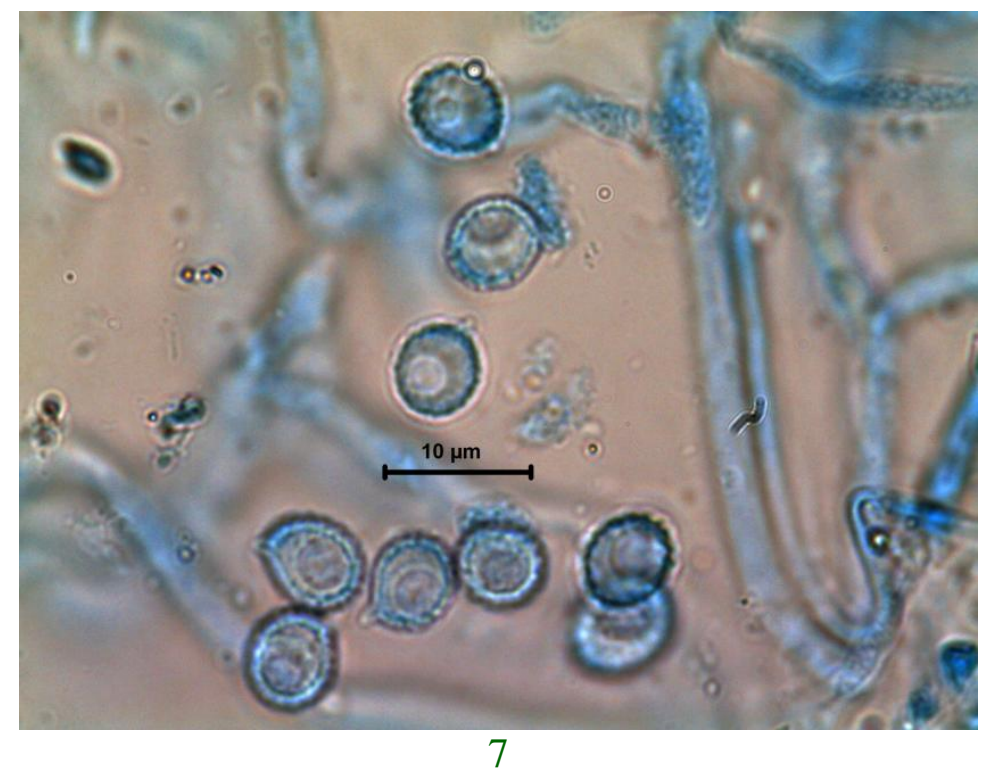

Fig.8-11 Russula congoana, 8-9 basidiocarp habit, 10 basidia and 11 basidiospores

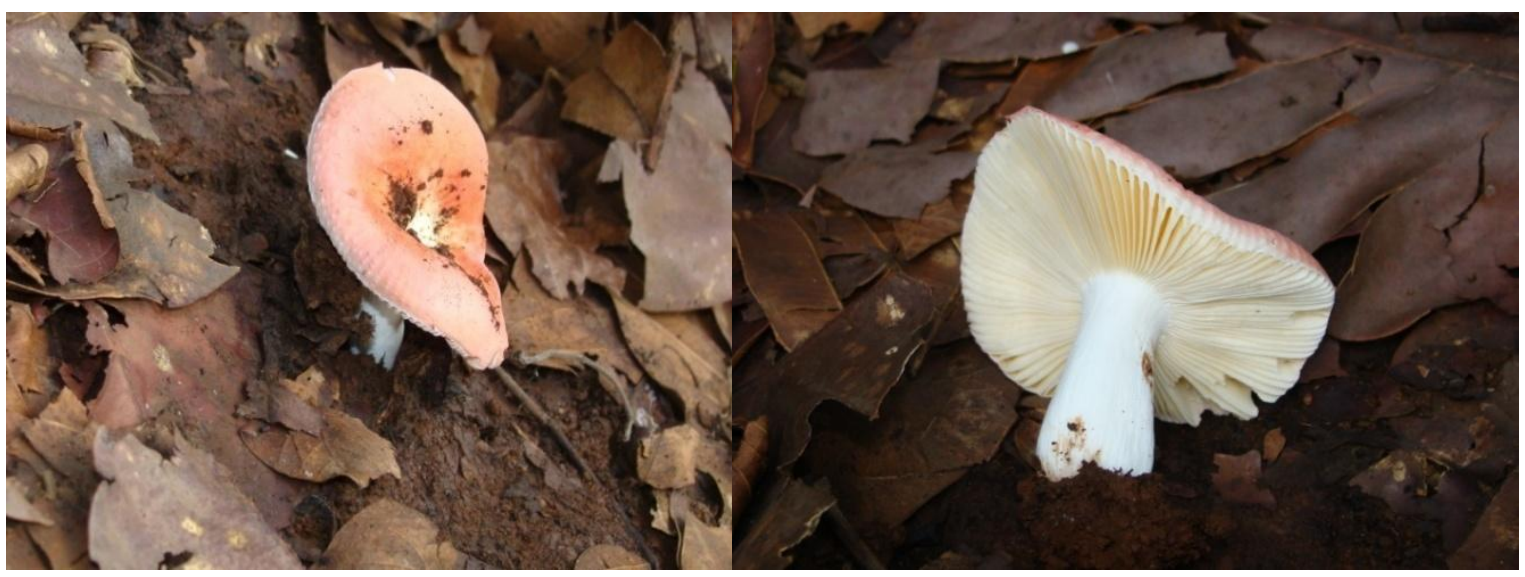

8

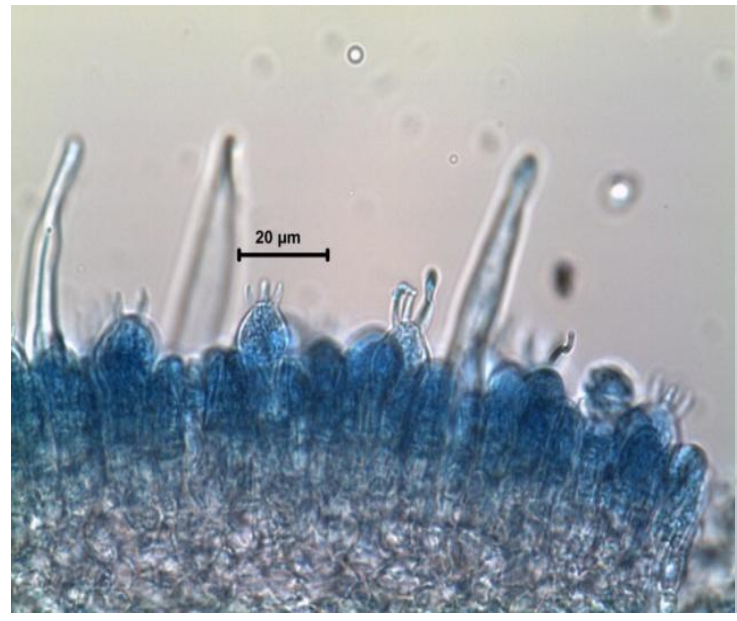

10

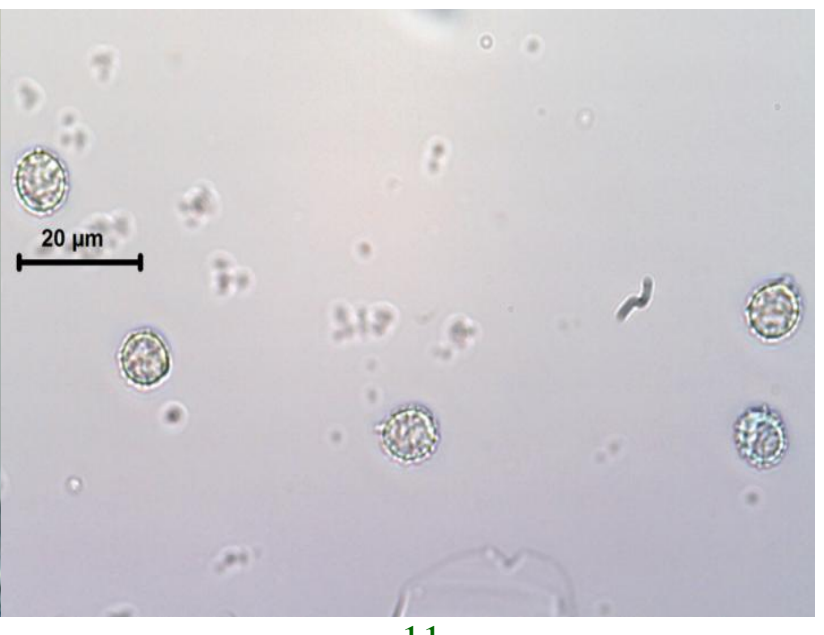

11 
Fig.12-14 Russula delicula, 12 basidiocarp, 13 basidia, 14 basidiospores

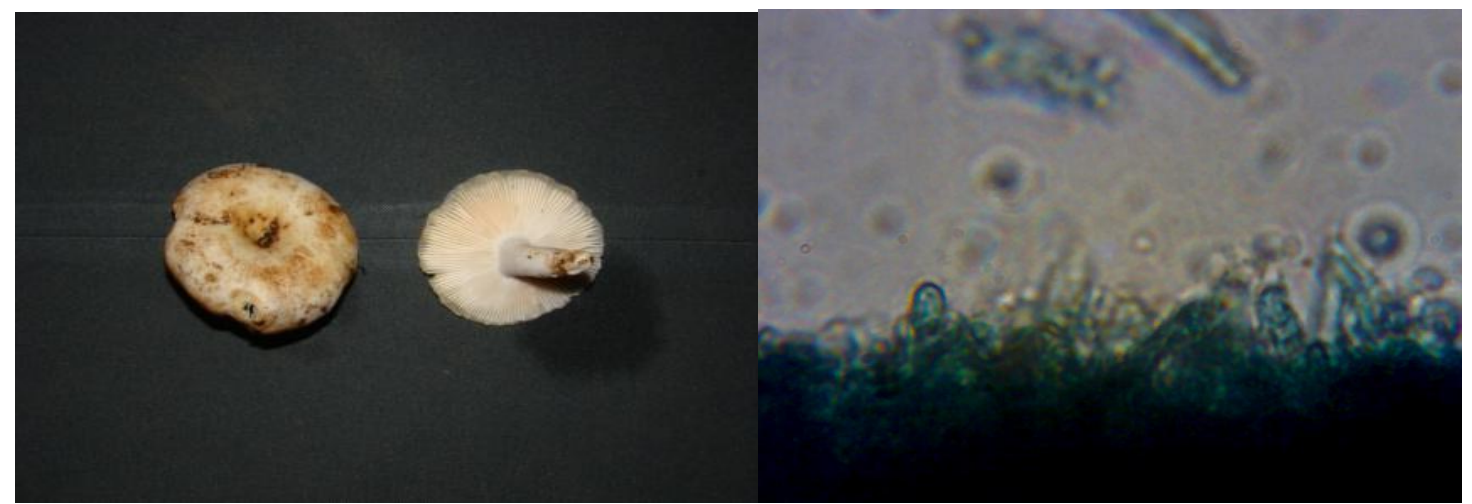

12

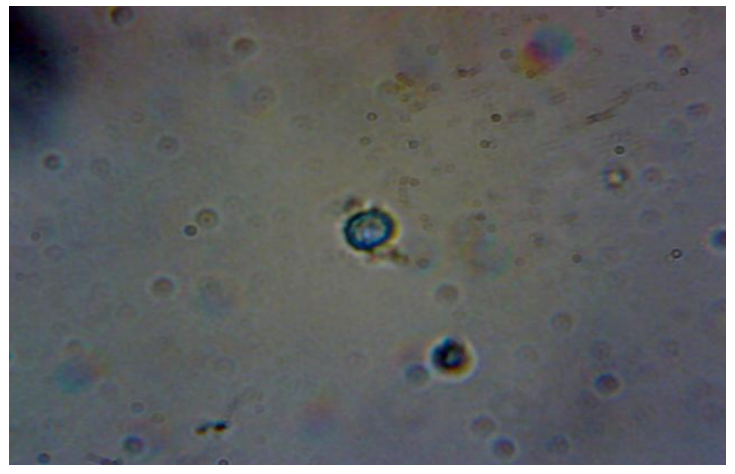

14

Fig.15-17 Russula leelavathyi, 15-16 basidiocarp, 17 basidia and basidiospores

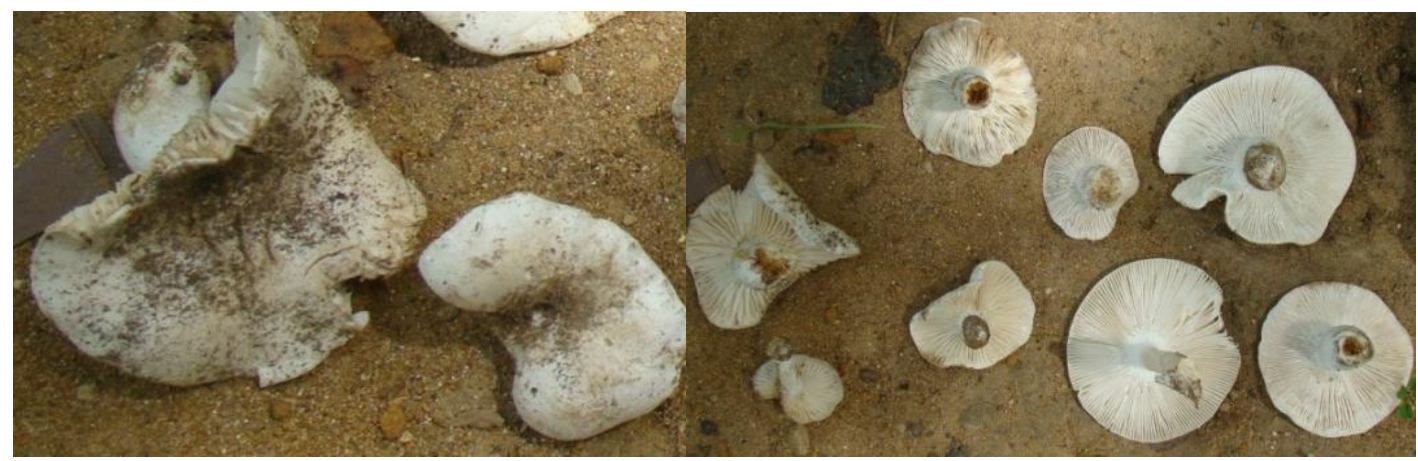

15 16

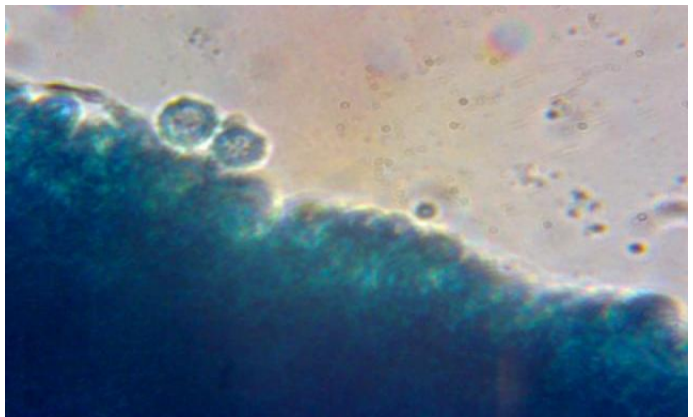


Fig.18-20 Russula michiganensis, 18 basidiocarps different developing statges, 19 tetrasporatebasidia with developing basidiospores, 20 basidiospores

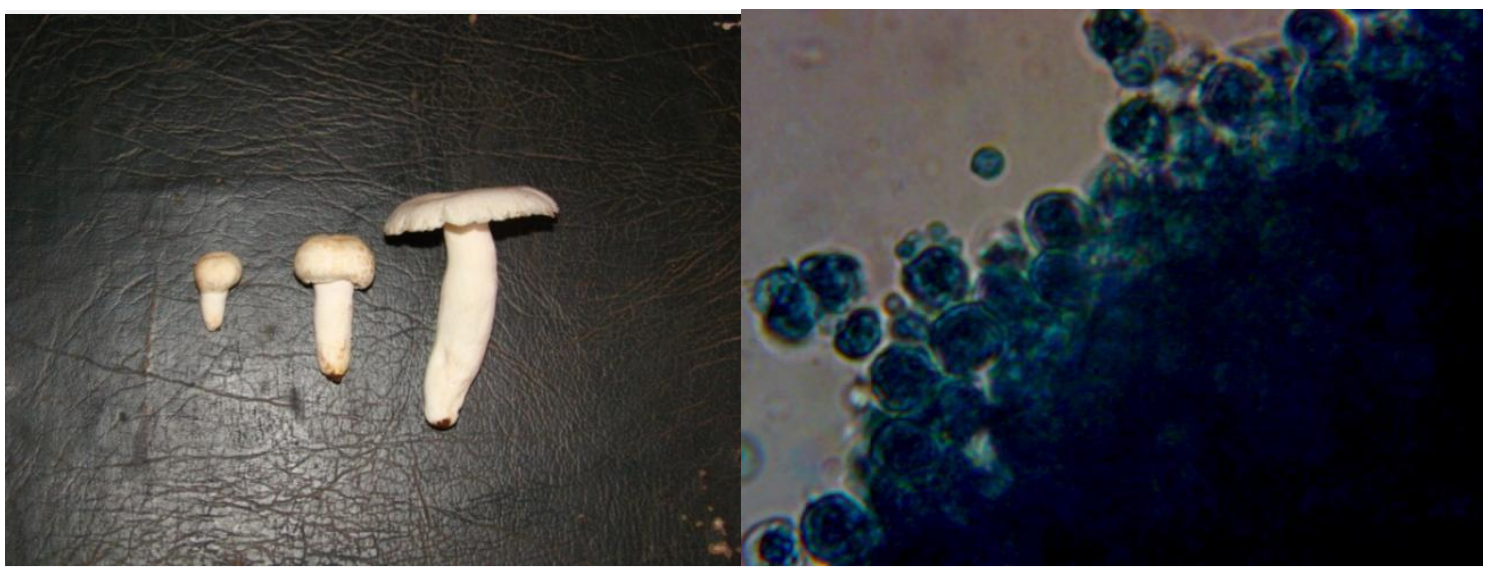

18

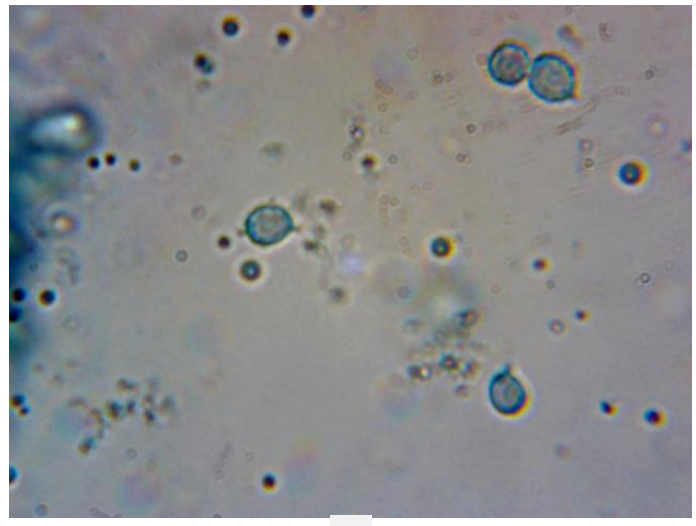

20

Fig.21 Tribal women selling Russula species in local market at Bajag in Dindori, Madhya Pradesh along with other mushroom (Astraeushygrometricus)

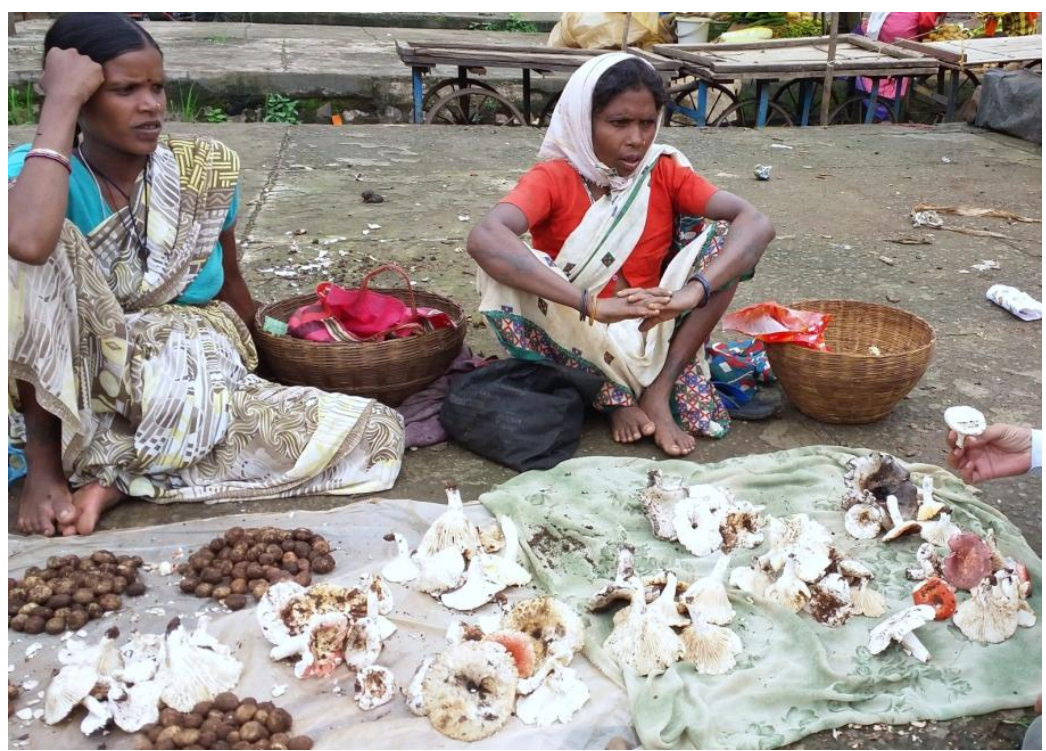


Lamellaeadnate, white turning grayish black to black on handling, more or less crowded with lamellulae of different lengths, with plenty of bifurcations; edge smooth, entire. Context white up to $5 \mathrm{~mm}$ thick. Discolouring blackish on exposure, heteromerous with nests of sphaerosytes. Stipe 3-6-5cm x 9$17 \mathrm{~mm}$ central, equal, solid; surface chalky white, turning black when handled. Annulus absent. Odour not distinctive. Spores 5-8 x 3$5 \mu \mathrm{m}$, subglobose to broadly ovoid ornamentation hardly up to $0.4 \mu \mathrm{m}$ high small to medium sized warts and thick and fine ridges are connected to form a partial reticulum; Suprahilar phage inamyloid. Basidiaclavate, 24-26 x 4-6 $\mu \mathrm{m}$, tetrasporate. Lamella-edge sterile with macrocystidia and

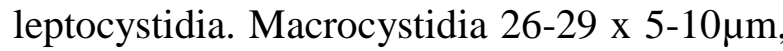
cylindric, thin-walled with refractive contents, concentrated near tip. Pleurocystidia abundant 27-55 x $4-7 \mu \mathrm{m}$ subcylindric with capitate apices or round with 2 or 3 sub-apical constriction, at times curved with scarcely visible contents, projecting prominently beyond the hymenium, very abundant. Subhymenium pseudoparenchymatous, hymenophoraltrama heteromorphous with sphaerocytes.

\section{Collection examined}

Mycorrhizal on sal, Amarkantak-Achanakmar Biosphere Reserve, Madhya Pradesh and Chhattisgarh, 24/07/2012, Mycology Herbarium, Tropical Forest Research Institute, Jabalpur, TF 2787.

Russula mushrooms distributd in different states of India are presented in Table 1. Total 124 species were recorded from 13 states. States-wise status of species is presented in Table 2. Edible species are presented in Table 3.

Russula adustaformed mycorrhizal association with sal (Shorearobusta Gaertn.) trees in central India. Its ectomycorrhizal association were reported in trees like, Hopeaponga, H. parviflora, Myristicamalabarica, Vateriaindica, and Diospyrosmalabarica. The species is distributed at different places in India: Kailana, Chakrata, Lohaghat, Mayawati, Jageshwar and Champawatin Uttarakhand (Saini and Atri 1984; Atri and Saini 1990b); Malappuram, Thiruvananthapuram in Kerala (Pradeep and Vrinda, 2010; Mohanan, 2011, 2014) and Mangalore in Karnataka (Pavithra et al., 2017). In the present study it is being reported from Amarkantak-Achanakmar Biosphere Reserve in Madhya Pradesh and Chhattisgarh, India.

Russula cinerella is being reported mycorrhizal with salfrom AmarkantakAchanakmar Biosphere Reserve, Madhya Pradesh and Chhattisgarh. It was also reported from Wayanad, Kerala growing solitary or scattered in large groups on soil under Hopeaparviflora, Vateriaindica, and Diospyrosmalabarica (Mohanan, 2011; 2014). The species is also reported to be distributed in Madagascar (Patouillard, 1924).

Russula congoana and $R$. deliculaform ectomycorrhizal asocoiarion with sal (Shorearobusta Gaertn.). R. congoana has worldwide distribution. It was earlier recorded from moist deciduous forests of India from Mussoorie, Uttarakhand (Sarwal 1984) and Ernakulam, Kollam, Malappuram, Wayanad and Thiruvananthapuram, Kerala. It is also distributed in moist-deciduous to evergreen forests and occurs solitary or scattered on soil under Hopeaponga, $H$. parviflora, Myristica malabarica, Vateriaindica, and Diospyros malabarica forming an ectomycorrhizal association. The species can be easily recognized by distinct pastel red pileus and the heavy ornamented the spores. Ir is an edible species (Pradeep and Vrinda 2010; Varghese et al., 2010; 
Mohanan 2011, 2014). R.deliculais widely distributedin moist deciduous forests of India and reported from Thiruvananthapuram and Wayanad, Kerala (Pradeep and Vrinda 2010; Mohanan 2011, 2014). Russula leelavathyi has worldwide distribution in North America, Europe andmoist deciduous forests of India it was reported growing on sandy soil and was associated with Hopeaparviflora from Thiruvananthapuram, Malappuram and Wayanad, Kerala (Vrinda et al., 1997a; Pradeep and Vrinda 2007, 2010; Mohanan 2011, 2014). In the present study it was also recorded from Amarkantaka-Achanakmarsal forest of Chhattisgarh and Madhya Pradesh.

Russula michiganensis is recorded on salfrom Amarkantaka-Achanakmarsal forest of Chhattisgarh and Madhya Pradesh. Earlier the species was recorded from moist deciduous forests of India forming ectomycorrhizal association with Hopeaponga, H. parviflora, Vateriaindica and Diospyrosmalabarica in Wayanad and Ernakulam, Kerala (Mohanan 2014). This mushroom has worldwide distribution. Russula senecis, an edible mushroom grows in association with Vateriaindica plants among the dipterocarp forests of Western Ghats (Natarajan et al., 2005), and in mixed forests in Sikkim (Das, 2009; Das et al., 2010) is collected from forests and sold in West Bengal (Khatua et al., 2015)

Many of Russula species are edible. Russula crustosais an edible mushroom reported from Shimla, Himachal Pradesh (Bhatt and Lakhanpal 1988a). R. luteais another edible mushroom growing in associated with Cedrusdeodara, Piceasmithiana, Pinuswalitiana, Quercusincana and Rhododendron arboreum in Shimla and lower Shiwalik Hills of Uttarakhand (Bhatt and Lakhanpal 1988b), another edible mushroom, $R$. lepida was recorded on humicolous soil from sal forests (Semwal et al., 2014). Other edible species include $R$. olivacea and $R$. parvovirescens growing in pine forest at Shillong in Meghalaya (Kalita et al., 2016). R.senecisis collected by tribes in West Bengal (Khatua et al., 2015).

In the present study out of six species reported some species for example, R.congoana collected from sal forest of Chhattisgarh and Madhya Pradesh are also edible, these mushrooms were collected by the local tribal people and sold in local market (Figure 21).

Mushrooms belong to genus Russula are widely distributed in India. 124 species of the genus are listed from 13 states. Uttarakhand represent the maximum diversity of Russula species and over $40 \%$ species recorded from this state only. Six species namely: Russula adusta, $R$. cinerella, $R$. congoana, $R$. delicula, $R$. leelavathyi and $R$. michiganensis are described occurring in sal forest of central India. These fungi form ectomycorrizal association with sal trees and some edible species are also collected by the local people and sold in thelocal market.

\section{Acknowledgements}

The authors are thankful to Dr. G. Rajeshwar Rao, Director, Tropical Forest Research Institute, Jabalpur for providing the research facilities. The work presented here was conducted under project ID No. 224/TFRI/2016/Patho-1(22) funded by India Council of Forest Research \& Education (ICFRE), Dehradun.

\section{References}

Abraham SP, Kachroo JL, Kaul TN (1980). Fleshy fungi of Gulmarg forest -I. Kavaka 8(1): 29-39.

Atri NS, Kour H (2003). Some unrecorded light spored mushrooms from Punjab. Indian Journal of Mushrooms 21:1-4. 
Atri NS, Saini SS (1990a) Studies on Russula Pers. Section Decolorantes (Maire) Sing. Geobios New Reports 9: 10-13.

Atri NS, Saini SS (1990b). North Indian Agaricales-VIII.The section compactae Fr. of Russula Pers. in India. Journal of the Indian Botanical Society 69, 343-346.

Atri NS, Saini SS (1990c). North Indian Agaricales-VIII. The Section Compactae Fr. of Russula Pers. Journal of Indian botanical Society 69: 343-346.

Atri NS, Saini SS, Gupta AK, Kaur A, Kour H, Saini SS (2010). Documentation of wild edible mushrooms and their seasonal availability in Punjab. In: Taxonomy and ecology of Indian fungi. (Eds.: Mukerji K.G. and Manoharachary C.) I.K. International Publishing House Pvt. Ltd. pp. 161-169.

Atri NS, Saini SS, Saini MK (1997). Studies on genus Russula Pers. from North Western Himalayas. Mushroom Res. 6: (1) 1-6.

Atri NS, Saini SS, Saini MK, Gupta AK (1992). Two new records of the genus Russula from India.Geobios new Reports 11: 101103.

Atri NS, Saini, SS (1986). Further contributions on the studies of northwest Himalayan Russula ceae. Geobios New Reports 5: 100-105.

Atri NS, Sharma Samidha, Kaur M, Das K (2016). Researches on Russula ceous Mushrooms - An Appraisal.Kavaka 47: 63-82.

Berkeley MJ (1851). Decades of fungi.Decades XXXII, XXXIII.Sikkim Himalaya fungi, collected by Dr. J.D. Hooker. Hooker's Journal of Botany and Kew Garden Miscellany 3: 39-49.

Berkeley MJ (1856). Decades of fungi.Decades I-LXII. Indian fungi. Hooker's London, Journal of Botany and Kew Garden Miscellany 8: 174-280.

Berkeley MJ (1876). Three fungi from Kashmir. Grevillea 4(31): 137-138.

Bhatt RP, Bhatt VK, Gaur RD (1995). Fleshy fungi of Gahwal Himalaya: The genus Russula. Indian Phytopathology 48: 402 411.
Bhatt RP, Lakhanpal TN (1988a). Russula crustosa Pk. - An addition to Indian edible mushrooms. Current Science 57(10): 560-561.

Bhatt RP, Lakhanpal TN (1988b). A new record of edible Russula from, India. Current Science 57(22): 1257-1258.

Bhatt RP, Purnima S, Semwal KC (2007). New records of Russula from Garhwal Himalaya. Mushroom Research 16(2): $55-60$.

Buyck Bart, Atri NS (2011). A Russula (Basidiomycota, Russula les) with an unprecedented hymenophore configuration from northwest Himalaya (India). Cryptogamie Mycologie. 32(2): 185-190.

ChaudharyRohina, TripathyAstha (2016). Diversity of wild mushroom in Himachal Pradesh (India). International Journal of Innovative Research in Science, Engineering and Technology 5(6): 1085910886.

Crous PW, Wingfield MJ, Burgess TI et al., (2017). Fungal Planet description sheets: 558-624. Persoonia 38: 240-384.

Crous PW, Wingfield MJ, Richardson DM et al., (2016). Fungal Planet description sheets: 400-468. Persoonia 36: 316-458

Das K (2009). Mushrooms of Sikkim I: Barsey Rhododendron Sanctuary. India: Botanical Survey of India \& Sikkim State Biodiversity Board.

Das K (2013). Diversity of ectomycorrhizalmacrofungi in Sikkim Himalaya with special reference to the genera Lactarius and Russula. In: Biodiversity and environmental issues (ed. MM Sheikh). LAP Lambert academic publishing, Germany, pp 224263.

Das K, Atri NS, Buyck B (2013a). Three new species of Russula (Russula les) from India.Mycosphere 4(4): 707-717.

Das K, Atri NS, Buyck B (2013b). Three new species of Russula (Russula les) from Sikkim (India). Mycosphere4(4): 722732. 
Das K, Dowie NJ, Li GJ, Miller SL (2014). Two new species of Russula (Russula les) from India. Mycosphere 5(5): 612-622.

Das K, Ghosh A, Chakraborty D et al., (2017). Fungal Biodiversity Profiles 31-40. CryptogamieMycologie38(3): 353-406.

Das K, Miller SL, Sharma JR (2006a). Russula in Himalaya 2: Four new taxa. Mycotaxon 95: 205-215.

Das K, Miller SL, Sharma JR, Hemenway J (2008). Two new species of Russula from Western Ghats in India.Indian Journal of Forestry 31(3), 473- 478.

Das K, Miller SL, Sharma JR, Sharma P, Bhatt RP (2005a). Russula in Himalaya I: A new species of subgenus Amoenula. Mycotaxon 94:85-88.

Das K, Sharma JR (2001b). Russula rhodomelaneaSarnari - a new record for India. Mushroom Research 10: 109-111.

Das K, Sharma JR (2003). New records of Russula from Kumaon Himalaya. Indian Journal of Forestry 26: 320-326.

Das K, Sharma JR (2005a). New records of Lactarius from India. Annals of Forestry 13: $1-8$.

Das K, Sharma JR (2005b). Russula ceae of Kumaon Himalaya.Botanical Survey of India, Ministry of Environment and Forests, Govt. of India, Kolkata, pp 255.

Das K, Sharma JR, Atri NS (2006b). Russula in Himalaya 3: A new species of subgenus Ingratula. Mycotaxon 95, 271-275.

Das K, Sharma JR, Bhatt RP (2002). Russula flavida Frost - an addition to the Indian ectomycorrhizic fungi. Mushroom Research 11: 9-10.

Das K, Sharma JR, Bhatt RP (2005). Two new varieties of Russula from India. Annals of Forestry 13: 281-286.

Das K, Van de Putte K, Buyck B (2010). New or interesting Russula from Sikkim Himalaya (India). Cryptogamie Mycologie 31(4): 373-387.

Das N, Mahapatra SC, Chattopadhyay RN (2002). Wild edible mushrooms: Nonwood forest products for livelihood generation. The Indian Forester 128 (4): 445-55. (2002)
Das, K. and Sharma, J.R. 2004b.Russula compacta Frost. \& peck- a new record from India. Journal of Mycology Plant Pathology 34:149-150.

Dhancholia S (2011). New records of genus Russula from dry temperate zone of Lahul Valley of Himachal Pradesh. Plant Disease Research 26: 203.

Dutta AK, Paloi S, Pradhan P, Acharya K (2015). A new species of Russula (Russula ceae) from India based on morphological and molecular (ITS sequence) data. Turkish Journal of Botany 39: 850-856.

Farook VA, Khan SS, Manimohan P (2013). A checklist of agarics (gilled mushrooms) of Kerala State, India.Mycosphere 4(1): 97131.

Florence EJM (2004). Biodiversity Documentation for Kerala, Part 2: Microorganisms (Fungi). KFRI Handbook No.17.Kerala Forest Research Institute, Peechi, Kerala, India.

Fries, E. (1838). Epicrisis SystematisMycologici. :1-610

Ghosh A, Das K (2017). Russula (Russula ceae) in western Himalaya 1: Two new species from subg. Russula.Phytotaxa 323(3): 337-252.

Ghosh A, Das K, Adhikari S, Bhatt RP (2016). A novel species of Russula (Russula ceae) from Indian Himalaya.Mycosphere 7(6): 771-785.

Ghosh A, Das K, Bhatt RP (2017). Russula sarnariisp. nov. (Russula ceae) from Indian Himalaya. Current Research in Environmental \& Applied Mycology 7(1): $64-72$.

Hedawoo GB (2010). Wild mushroom flora from Amravati Regoin, Maharastra, India. Journal of Mycology and Plant Pathology 40(3): 441-444.

Joshi S, Bhatt RP, Stephenson SL (2012). The current status of the family Russula ceae in the Uttarakhand Himalaya, India. Mycosphere 3(4): 486-501.

Kalita K, Bezbaroa RN, Kumar R, Pandey S (2016). Documentation of wild edible mushrooms from Meghalaya, Northeast 
India. Current Research in Environmental \& Applied Mycology 6(3): 238-247.

Kaur M, Atri NS, Sharma S, Singh Y (2011). Three taxa of genus Russula Pers section Tenellae from India. Journal of Mycology and Plant Pathology 41(4): 524-527.

Khatua S, Dutta AK, Acharya K. (2015). Prospecting Russula senecis: A delicacy among the tribes of West Bengal. PeerJPrePrints 3:e707v2

Kumar R, Tapwal A, Pandey S, Raja-Rishi R, Mishra G, Giri K. (2014). Six unrecorded species of Russula (Russula les) from Nagaland, India and their nutrient composition. Nusantara Bioscience 6(1): 33-38

Manimohan P, DeepnaLatha KP (2011). Observations on two rarely collected species of Russula.Mycotaxon 116: 125131.

Mohanan C (2011). Macrofungi of Kerala.Kerala Forest Research Institute, Hand Book \# 27, Kerala, India, 597pp.

Mohanan C (2014). Macrofungal diversity in the Western Ghats, Kerala, India: members of Russula ceae. Journal of Threatened Taxa 6(4): 5636-5648.

Mundkur BB (1938a). Fungi of India.Suppliment I. ICAR Science Monograph 12: 54p.

Natarajan K, Raman N (1983). South Indian Agaricales. J. Cramer, Vaduz, Germany.

Natarajan K, Senthilarsu G, Kumaresan V, Riviere $\mathrm{T}$ (2005). Diversity in ectomycorrhizal fungi of a dipterocarp forest in Western Ghats. Current Science. 88:1893-1895.

Paloi S, Dutta AK, Acharya K (2015). A new species of Russula (Russula les) from Eastern Himalaya, India.Phytotaxa 234(3):255-262.

Paloi S, Dutta AK, Pradhan P, Roy A, Acharya K (2016). Russula buyckii, a new species of Russula subgenus Incrutatula from Eastern Himalaya, India. Phytotaxa 252(2): 123-130.

Patouillard NT (1914). Quelques Champignons $\mathrm{du}$ Congo. Bulletin de la
SociétéMycologique de France 30(3): 336-346.

Patouillard, N.T. (1924). Basidiomycetes nouveaux de Madagascar. Bulletin du Muséumd'Histoire Naturelle Paris. 30:526-532.

Pavithra M, Sridhar KR, Greeshma AA (2017). Macrofungi in two botanical gardens in southwestern India. Journal of Threatened Taxa 9(3): 9962-9970.

Pradeep CK, Vrinda KB (2007). Some noteworthy agarics from Western Ghats of Kerala. Journal of Mycopathological Research 45(1): 1-14.

Pradeep CK, Vrinda KB (2010). Ectomyrrhizal fungal diversity in three types and their association with endemic, indigenous and exotic species in the Western Ghar forests of Thiruvanthapuram districtm Kerala. Journal of Mycopathological Research 48(2): 279-289.

Rawla GS (2001). Himalayan Species of Russula Pers. Ex S.F. Gray, 1-48. In: Plant Diversity of the Himalaya (eds. PC Pande, SS Samant). GyanodayaPrakashan, Nainital, India, pp $1-48$.

Rawla GS, Sarwal BM (1983). Taxonomic studies on Indian Agarics 1. Russula ceae. Bibliotheca Mycologica 91: 23-46.

Romagnesi, H. (1945). Contribution à l'étude des Russules de la florefrançaise (3ième série). Bulletin de la SociétéMycologique de France. 61:22-37.

Saini MK, Atri NS, Sharma Samidha, PriyaJanki (2010). Taxonomic Studies on the Genus Russula Pers. from Himachal Pradesh, India. Journal of Mycology and Plant Pathology 40 (1): 52-54.

Saini SS, Atri NS (1981). Russula foetens (Pers.) Fr. - A new record for India. Current Science 10: 460-461.

Saini SS, Atri NS (1984). Studies on the NorthWest Himalayan Russula ceae. Geobios New Reports 3: 4-6.

Saini SS, Atri NS (1989a). North Indian Agaricales-IX section Ingratae Quel. Of Russula Pers. Kavaka 17: 21-27. 
Saini SS, Atri NS (1989b). North Indian Agaricales XI-Section Russula Pers. in India, Indian Journal of Mycology and Plant Pathology 19: 44-49.

Saini SS, Atri NS, Bhupal M (1988). North Indian Agaricales V.Indian Phytopathology 41: 622-625.

Saini SS, Atri NS, Saini MK (1989). North Indian Agaricales VI. Journal of the Indian Botanical Society 68: 205-208.

Sarwal BM (1984). Taxonomic studies on Indian Agarics II. Indian Phytopathology 37: 228-233.

Sathe AV, Deshpande S, Kulkarni SM, Daniel J (1980) (1981). Agaricales (Mushrooms) of South-west India - Part I: Agaricales (Mushrooms) of Maharashtra State (MACS, Pune). Pp.1-114.

Semwal KC, Stephenson SL, Bhatt VK, Bhatt RP (2014). Edible mushrooms of the Northwestern Himalaya, India: A study of indigenous knowledge, distribution and diversity. Mycosphere 5(3): 440-461.

Shaffer R.L. (1962). The subsection Compactae of Russula. Brittonia 14(3): 254-284.
Shajahan M, Samajpati N (1995). Ectomycorrhizal fungi of Shorearobusta G.G. from West Bengal. Indian Journal of Mycological Research 33: 105-117.

Sharma JR, Das K (2002). New records of Russula ceae from India. Phytotaxonomy 2: 11-15.

Varghese SP, Pradeep CK, Vrida KB (2010). Mushrooms of tribal importance in Wayanad area of Kerala. Journal of Mycopathological Research 48(2): 311320.

Vishwakarma MP, Bhatt RP, Joshi Sweta (2012). Macrofungal diversity in moist temperate forests of Garhwal Himalaya. Indian Journal of Science and Technology 5(1): 1928-1932.

Vrinda KB, Pradeep CK and Abraham TK (1997c). A new species of Russula from Kerala, India. Mycotaxon 62: 87-96.

Vrinda, K.B.; Pradeep, C.K.; Abraham, T.K. (1997). A new species of Russula from Kerala, India. Mycotaxon 62: 389-394.

Watling R and Gregory NM (1980). Larger fungi from Kashmir. Nova Hedwigia 32(2-3): 493-564.

\section{How to cite this article:}

Verma, R.K., Vimal Pandro and Abhishek Pyasi. 2018. Diversity and Distribution of Russula in India with Reference to Central Indian Species. Int.J.Curr.Microbiol.App.Sci. 7(10): 3078-3103. doi: https://doi.org/10.20546/ijcmas.2018.710.359 